\title{
Mixed Finite Element Methods for Quasilinear Second-Order Elliptic Problems*
}

\author{
By F. A. Milner
}

\begin{abstract}
A mixed finite element method is developed to approximate the solution of a quasilinear second-order elliptic partial differential equation. The existence and uniqueness of the approximation are demonstrated and optimal rate error estimates are derived.
\end{abstract}

1. Introduction. Let $\Omega \subset \subset \mathbf{R}^{2}$ be a domain with $C^{2}$ boundary $\partial \Omega$. We shall assume that for some $\varepsilon, 0<\varepsilon<1$, and for each pair of functions $(f, g)$ in $H^{\varepsilon}(\Omega) \times$ $H^{3 / 2+\varepsilon}(\partial \Omega)$ there exists a unique solution $p \in H^{2+\varepsilon}(\Omega)$ of the quasilinear Dirichlet problem

(a) $L p=-\nabla \cdot(a(p) \nabla p+\mathbf{b}(p))+c(p)=f$ in $\Omega$,

(b) $p=-g$ on $\partial \Omega$,

where $\nabla w$ denotes the gradient of a scalar function $w$, and $\nabla \cdot \mathbf{v}$ denotes the divergence of a vector function $\mathbf{v}$. Note that then $p$ belongs to $W^{1, \infty}(\Omega)$, which will be needed throughout the paper.

We shall also assume that the coefficients $a: \bar{\Omega} \times \mathbf{R} \rightarrow \mathbf{R}, \mathbf{b}: \bar{\Omega} \times \mathbf{R} \rightarrow \mathbf{R}^{2}$, and $c$ : $\overline{\mathbf{\Omega}} \times \mathbf{R} \rightarrow \mathbf{R}$ are twice continuously differentiable with bounded derivatives through second order; moreover, assume that $a(x, q) \geqslant a_{1}>0$. The variable $x$ will normally be omitted in this notation below.

For $1 \leqslant s<\infty$ and $k$ any nonnegative integer let

$$
W^{k, s}(\Omega)=\left\{f \in L^{s}(\Omega) \mid D^{\alpha} f \in L^{s}(\Omega) \text { if }|\alpha| \leqslant k\right\}
$$

denote the Sobolev space endowed with the norm

$$
\|f\|_{k, s ; \Omega}=\left(\sum_{|\alpha| \leqslant k}\left\|D^{\alpha} f\right\|_{L^{s}(\Omega)}^{s}\right)^{1 / s}
$$

(the subscript $\Omega$ will always be omitted unless necessary to avoid ambiguity). Let $H^{k}(\Omega)=W^{k, 2}(\Omega)$ with norm $\|\cdot\|_{k}=\|\cdot\|_{k, 2}$ (the notation $\|\cdot\|_{0}$ will mean $\|\cdot\|_{L^{2}(\Omega)}$ or $\left.\|\cdot\|_{L^{2}(\Omega)^{2}}\right)$. For $0 \leqslant r<\infty$ let $W^{r, s}(\Omega), W^{r, s}(\partial \Omega), H^{r}(\Omega)$, and $H^{r}(\partial \Omega)$ denote the fractional order Sobolev spaces with norms $\|\cdot\|_{r, s ; \Omega},\|\cdot\|_{r, s ; \partial \Omega},\|\cdot\|_{r ; \Omega}$, and $\|\cdot\|_{r: \partial \Omega}$.

Received August 3, 1983; revised April 10, 1984.

1980 Mathematics Subject Classification. Primary 65N15, 65N30; Secondary 41A10, 41A25.

*This paper represents work done by the author for his $\mathrm{Ph}$. D. thesis at the University of Chicago, and was partly supported by the Aileen S. Andrew Foundation. 
We shall denote by $($,$) the inner product in either L^{2}(\Omega)$ or $L^{2}(\Omega)^{2}$, that is,

$$
(\eta, \theta)=\int_{\Omega} \eta \theta d x \text { or } \int_{\Omega} \eta \cdot \theta d x
$$

Let $\langle$,$\rangle be the L^{2}$-inner product on the boundary of $\Omega$ :

$$
\langle\lambda, \pi\rangle=\int_{\partial \Omega} \lambda \pi d s .
$$

We shall use the same notations to indicate the dualities between $W^{r, s}(\Omega)$ and $W^{r, s}(\Omega)^{\prime}$ and $H^{s}(\partial \Omega)$ and $H^{-s}(\partial \Omega)$, respectively.

Let

$$
\mathbf{V}=\mathbf{H}(\operatorname{div} ; \Omega)=\left\{\mathbf{v} \in L^{2}(\Omega)^{2} \mid \operatorname{div} \mathbf{v} \in L^{2}(\Omega)\right\}
$$

normed by

$$
\|\mathbf{v}\|_{\mathbf{v}}=\|\mathbf{v}\|_{0}+\|\operatorname{div} \mathbf{v}\|_{0}
$$

and let

$$
\mathbf{H}^{s}(\operatorname{div} ; \Omega)=\left\{\mathbf{v} \in L^{2}(\Omega)^{2} \mid \operatorname{div} \mathbf{v} \in H^{s}(\Omega)\right\}
$$

normed by

$$
\|\mathbf{v}\|_{\mathbf{H}^{s}(\operatorname{div} ; \Omega)}=\|\mathbf{v}\|_{0}+\|\operatorname{div} \mathbf{v}\|_{s}
$$

Let

$$
W=L^{2}(\Omega)
$$

If

$$
\mathbf{u}=-(a(p) \nabla p+\mathbf{b}(p)), \quad \alpha=1 / a, \quad \boldsymbol{\beta}=\alpha \mathbf{b},
$$

then $(\mathbf{u}, p) \in \mathbf{V} \times W$ is a solution of the following weak formulation of (1.1):

$$
\begin{aligned}
& \text { (a) }(\alpha(p) \mathbf{u}, \mathbf{v})-(\operatorname{div} \mathbf{v}, p)+(\boldsymbol{\beta}(p), \mathbf{v})=\langle g, \mathbf{v} \cdot \mathbf{v}\rangle, \quad \mathbf{v} \in \mathbf{v}, \\
& \text { (b) }(\operatorname{div} \mathbf{u}, w)+(c(p), w)=(f, w), \quad w \in W,
\end{aligned}
$$

where $v$ denotes the unit outward normal vector to $\partial \Omega$. Since $\mathbf{v} \cdot v \in H^{-1 / 2}(\partial \Omega)$ (see [12], [16]), the duality $\langle g, \mathbf{v} \cdot v\rangle$ is well-defined.

Mixed finite element methods for (1.1) are discrete versions of (1.3) and have been treated for linear operators $L$ by several authors [2], [5]-[7], [9], [12]-[16].

Let $\mathscr{T}_{h}$ be a quasi-regular polygonalization of $\Omega$ (by triangles, rectangles, or possibly parallelograms), with boundary polygons allowed to have one curved side, of characteristic parameter $h \in(0,1)$, and let

$$
\mathbf{V}_{h} \times W_{h} \subset \mathbf{V} \times W
$$

be the associated Raviart-Thomas-Nedelec space of index $k \geqslant 0$, [11], [12]. To be more explicit, for $E \subset \mathbf{R}^{2}$ let $P_{k}(E)$ denote the restrictions of polynomials of total degree $k$ to the set $E$ and let $Q_{k}(E)$ denote the restriction of $P_{k}(\mathbf{R}) \otimes P_{k}(\mathbf{R})$ to $E$. Then, let $R_{k}(E)=P_{k}(E)$ if $E$ is a triangle (interior or boundary) and $R_{k}(E)=$ $Q_{k}(E)$ if $E$ is a rectangle (interior or boundary), and let $\mathbf{R}_{k}(E)=R_{k}(E)^{2}$. For any $E \in \mathscr{T}_{h}$ let

$$
\mathbf{V}(E)=\mathbf{R}_{k}(E) \oplus \operatorname{Span}\left\{\mathbf{x} R_{k}(E)\right\}, \quad W(E)=R_{k}(E)
$$


Set

$$
\begin{aligned}
\mathbf{V}_{h} & =\mathbf{V}\left(k, \mathscr{T}_{h}\right)=\left\{\mathbf{v} \in \mathbf{V}|\mathbf{v}|_{E} \in \mathbf{V}(E), E \in \mathscr{T}_{h}\right\} \\
& =\left\{\mathbf{v} \in \prod_{E \in \mathscr{T}_{h}} \mathbf{V}(E)|\mathbf{v}|_{E_{i}} \cdot v_{i}+\left.\mathbf{v}\right|_{E_{i}} \cdot v_{j}=0 \text { on } \bar{E}_{i} \cap \bar{E}_{j}\right\},
\end{aligned}
$$

where $v_{l}, l=i, j$, is the outer normal to $\partial E_{l}$ on $\bar{E}_{i} \cap \bar{E}_{j}$; also, let

$$
W_{h}=W\left(k, \mathscr{T}_{h}\right)=\left\{w \in W|w|_{E} \in W(E), E \in \mathscr{T}_{h}\right\} .
$$

Let $\pi_{h}: \mathbf{V} \rightarrow \mathbf{V}_{h}$ be the Raviart-Thomas projection, [6], [12], which satisfies (see [13] for $q \neq 2$ below)

$$
\begin{gathered}
\left(\operatorname{div}\left[\pi_{h} \mathbf{v}-\mathbf{v}\right], w\right)=0, \quad \mathbf{v} \in \mathbf{V}, w \in W_{h}, \\
\text { (1.5) }\left\|\pi_{h} \mathbf{v}-\mathbf{v}\right\|_{0, q} \leqslant Q\|\mathbf{v}\|_{s, q} h^{s}, \quad 1 / q<s \leqslant k+1 \text {, if } \mathbf{v} \in \mathbf{V} \cap W^{s, q}(\Omega)^{2}, \\
\text { (1.6) }\left\|\operatorname{div}\left(\pi_{h} \mathbf{v}-\mathbf{v}\right)\right\|_{0} \leqslant Q\|\operatorname{div} \mathbf{v}\|_{s} h^{s}, \quad 0 \leqslant s \leqslant k+1 \text {, if } \mathbf{v} \in \mathbf{V} \cap \mathbf{H}^{s}(\operatorname{div} ; \Omega) .
\end{gathered}
$$

Let $P_{h}: W \rightarrow W_{h}$ be the orthogonal $L^{2}$-projection into $W_{h}$ defined by

$$
\left(P_{h} w-w, \chi\right)=0, \quad w \in W, \chi \in W_{h},
$$

which satisfies

$$
\begin{gathered}
\left\|P_{h} w-w\right\|_{0, q} \leqslant Q\|w\|_{s, q} h^{s}, \quad 0 \leqslant s \leqslant k+1, \text { if } w \in W \cap W^{s, q}(\Omega), \\
\left\|P_{h} w-w\right\|_{-r} \leqslant Q\|w\|_{s} h^{r+s}, \quad 0 \leqslant r, s \leqslant k+1, \text { if } w \in H^{s}(\Omega),
\end{gathered}
$$

We can now formulate the mixed finite element method to approximate the solution of (1.1):

Find $\left(\mathbf{u}_{h}, p_{h}\right) \in \mathbf{V}_{h} \times W_{h}$ such that

$$
\begin{aligned}
& \text { (a) }\left(\alpha\left(p_{h}\right) \mathbf{u}_{h}, \mathbf{v}\right)-\left(\operatorname{div} \mathbf{v}, p_{h}\right)+\left(\boldsymbol{\beta}\left(p_{h}\right), \mathbf{v}\right)=\langle g, \mathbf{v} \cdot v\rangle, \quad \mathbf{v} \in \mathbf{V}_{h}, \\
& \text { (b) }\left(\operatorname{div} \mathbf{u}_{h}, w\right)+\left(c\left(p_{h}\right), w\right)=(f, w), \quad w \in W_{h} .
\end{aligned}
$$

We shall demonstrate in Section 2 the existence of a solution $\left(\mathbf{u}_{h}, p_{h}\right) \in \mathbf{V}_{h} \times W_{h}$ of the nonlinear algebraic system (1.11) through an adaptation of the method used by Douglas in [4]. In Section 4, we shall establish the uniqueness of that solution inside a certain ball. Furthermore, we shall show that $\left(\mathbf{u}_{h}, p_{h}\right)$ converges to $(\mathbf{u}, p)$ in $L^{2}(\Omega)^{2} \times L^{2}(\Omega)$ at an optimal rate as $h \downarrow 0$ (Section 3$)$ and also in $\left(H^{\mathrm{s}}(\Omega)^{2}\right)^{\prime} \times$ $H^{s}(\Omega)^{\prime}, 0<s \leqslant k+1$, provided that the boundary of $\Omega$, the coefficients $a$, b, and $c$, and the solution $p$ of (1.1) are smooth enough (Section 6). In Section 5, we establish the convergence of $p_{h}$ to $p$ in $L^{q}(\Omega), 2 \leqslant q \leqslant \infty$, at an optimal rate as $h \rightarrow 0$.

2. Solvability of the Discrete Problem. For $\rho \in W_{h}$, we shall write

$$
\alpha(\rho)-\alpha(p)=-\tilde{\alpha}_{p}(\rho)(p-\rho)=-\alpha_{p}(p)(p-\rho)+\tilde{\alpha}_{p p}(\rho)(p-\rho)^{2}
$$

where

$$
\begin{aligned}
\tilde{\alpha}_{p}(\rho) & =\int_{0}^{1} \alpha_{p}(\rho+t[p-\rho]) d t \\
\tilde{\alpha}_{p p}(\rho) & =\int_{0}^{1}(1-t) \alpha_{p p}(p+t[\rho-p]) d t
\end{aligned}
$$

are bounded functions in $\bar{\Omega}$. Similarly, we can write

$$
\boldsymbol{\beta}(\rho)-\boldsymbol{\beta}(p)=-\boldsymbol{\beta}_{p}(p)(p-\rho)+\tilde{\boldsymbol{\beta}}_{p p}(\rho)(p-\rho)^{2}=-\tilde{\boldsymbol{\beta}}_{p}(\rho)(p-\rho),
$$




$$
c(\rho)-c(p)=-c_{p}(p)(p-\rho)+\tilde{c}_{p p}(\rho)(p-\rho)^{2}=-\tilde{c}_{p}(\rho)(p-\rho),
$$

where $\tilde{\beta}_{p}(\rho), \tilde{c}_{p}(\rho), \tilde{\boldsymbol{\beta}}_{p p}(\rho)$, and $\tilde{c}_{p p}(\rho)$ are bounded functions in $\bar{\Omega}$.

If we now subtract (1.11) from (1.3), we obtain the error equations

(a) $\left(\alpha(p)\left[\mathbf{u}-\mathbf{u}_{h}\right], \mathbf{v}\right)-\left(\operatorname{div} \mathbf{v}, p-p_{h}\right)+\left(\boldsymbol{\beta}_{p}(p)\left[p-p_{h}\right], \mathbf{v}\right)$

$$
=\left(\left[\boldsymbol{\alpha}\left(p_{h}\right)-\alpha(p)\right] \mathbf{u}_{h}+\boldsymbol{\beta}\left(p_{h}\right)-\boldsymbol{\beta}(p)+\boldsymbol{\beta}_{p}(p)\left[p-p_{h}\right], \mathbf{v}\right),
$$

(b) $\left(\operatorname{div}\left[\mathbf{u}-\mathbf{u}_{h}\right], w\right)+\left(c_{p}(p)\left[p-p_{h}\right], w\right)$

$$
\mathbf{v} \in \mathbf{V}_{h} \text {, }
$$

$$
=\left(c\left(p_{h}\right)-c(p)+c_{p}(p)\left[p-p_{h}\right], w\right), \quad w \in W_{h} .
$$

Substituting (2.1), (2.2), and (2.3) into (2.4), we see that (with $\rho=p_{h}$ )

(a) $\quad\left(\alpha(p)\left[\mathbf{u}-\mathbf{u}_{h}\right], \mathbf{v}\right)-\left(\operatorname{div} \mathbf{v}, p-p_{h}\right)+\left(\left[\alpha_{p}(p) \mathbf{u}+\boldsymbol{\beta}_{p}(p)\right]\left(p-p_{h}\right), \mathbf{v}\right)$

$$
=\left(\left[\tilde{\alpha}_{p p}\left(p_{h}\right) \mathbf{u}+\tilde{\boldsymbol{\beta}}_{p p}\left(p_{h}\right)\right]\left(p-p_{h}\right)^{2}+\tilde{\boldsymbol{\alpha}}_{p}\left(p_{h}\right)\left(p-p_{h}\right)\left(\mathbf{u}-\mathbf{u}_{h}\right), \mathbf{v}\right),
$$

$$
\mathbf{v} \in \mathbf{V}_{h} \text {, }
$$

(b) $\left(\operatorname{div}\left[\mathbf{u}-\mathbf{u}_{h}\right], w\right)+\left(c_{p}(p)\left[p-p_{h}\right], w\right)=\left(\tilde{c}_{p p}\left(p_{h}\right)\left[p-p_{h}\right]^{2}, w\right)$,

$$
w \in W_{h} \text {. }
$$

Set $\Gamma=\alpha_{p}(p) \mathbf{u}+\boldsymbol{\beta}_{p}(p) \in C_{B}^{0}(\Omega)$ and $\gamma=c_{p}(p) \in C_{B}^{1}(\Omega)$. Let us now replace $\mathbf{u}$ by $\pi_{h} \mathbf{u}$ and $p$ by $P_{h} p$ on the left-hand side of (2.5) to obtain (using (1.4) and (1.10)) the relations

(a) $\left(\alpha(p)\left[\pi_{h} \mathbf{u}-\mathbf{u}_{h}\right], \mathbf{v}\right)-\left(\operatorname{div} \mathbf{v}, P_{h} p-p_{h}\right)+\left(\Gamma\left[P_{h} p-p_{h}\right], \mathbf{v}\right)$

$$
\begin{array}{r}
=\left(\alpha(p)\left[\pi_{h} \mathbf{u}-\mathbf{u}\right]+\Gamma\left[P_{h} p-p\right]+\left[\tilde{\alpha}_{p p}\left(p_{h}\right) \mathbf{u}+\tilde{\boldsymbol{\beta}}_{p p}\left(p_{h}\right)\right]\left(p-p_{h}\right)^{2}\right. \\
\left.+\tilde{\alpha}_{p}\left(p_{h}\right)\left(p-p_{h}\right)\left(\mathbf{u}-\mathbf{u}_{h}\right), \mathbf{v}\right), \\
\mathbf{v} \in \mathbf{V}_{h},
\end{array}
$$

(b) $\left(\operatorname{div}\left[\pi_{h} \mathbf{u}-\mathbf{u}_{h}\right], w\right)+\left(\gamma\left[P_{h} p-p_{h}\right], w\right)$

$$
=\left(\gamma\left[P_{h} p-p\right]+\tilde{c}_{p p}\left(p_{h}\right)\left(p-p_{h}\right)^{2}, w\right), \quad w \in W_{h} .
$$

Now let $M: H^{2}(\Omega) \rightarrow L^{2}(\Omega)$ be the operator

$$
M w=-\nabla \cdot(a(p) \nabla w+a(p) \Gamma w)+\gamma w,
$$

and let $M^{*}$ be its formal adjoint; that is,

$$
M^{*} \chi=-\nabla \cdot(a(p) \nabla \chi)+a(p) \Gamma \cdot \nabla \chi+\gamma \chi .
$$

We shall assume that the restrictions of $M$ and $M^{*}$ to $H^{2}(\Omega) \cap H_{0}^{1}(\Omega)$ have bounded inverses; that is, for any $\psi \in L^{2}(\Omega)$ there exists a unique $\phi \in H^{2}(\Omega) \cap$ $H_{0}^{1}(\Omega)$ such that $M \phi=\psi$ (respectively, $M^{*} \phi=\psi$ ) and $\|\phi\|_{2} \leqslant Q\|\psi\|_{0}$. This would be guaranteed by assuming $c_{p} \geqslant 0$ (see, for example, [8]). Let

$$
\Phi: \mathbf{V}_{h} \times W_{h} \rightarrow \mathbf{V}_{h} \times W_{h}
$$


be given by $\Phi((\mu, \rho))=(y, z),(y, z)$ being the (unique) solution of the system

(a) $\left(\alpha(p)\left[\pi_{h} \mathbf{u}-\mathbf{y}\right], \mathbf{v}\right)-\left(\operatorname{div} \mathbf{v}, P_{h} p-z\right)+\left(\Gamma\left[P_{h} p-z\right], \mathbf{v}\right)$

$$
\begin{array}{r}
=\left(\alpha(p)\left[\pi_{h} \mathbf{u}-\mathbf{u}\right]+\Gamma\left[P_{h} p-p\right]+\left[\tilde{\boldsymbol{\alpha}}_{p p}(\rho) \mathbf{u}+\tilde{\boldsymbol{\beta}}_{p p}(\rho)\right](p-\rho)^{2}\right. \\
\left.+\tilde{\alpha}_{p}(\rho)(p-\rho)(\mathbf{u}-\mu), \mathbf{v}\right), \\
\mathbf{v} \in \mathbf{V}_{h},
\end{array}
$$

(b) $\left(\operatorname{div}\left[\pi_{h} \mathbf{u}-\mathbf{y}\right], w\right)+\left(\gamma\left[P_{h} p-z\right], w\right)$

$$
=\left(\gamma\left[P_{h} p-p\right]+\tilde{c}_{p p}(\rho)(p-\rho)^{2}, w\right), \quad w \in W_{h},
$$

the existence of which follows for small $h$ from [5], since the left-hand side of (2.8) corresponds to the mixed method for the operator $M$. Thus, $(\mathbf{y}, z)$ is the solution of a linear algebraic system of the form

$$
\begin{gathered}
(\alpha(p) \psi, \mathbf{v})-(\operatorname{div} \mathbf{v}, \phi)+(\Gamma \phi, \mathbf{v})=F(\mathbf{v}), \quad \mathbf{v} \in \mathbf{V}_{h}, \\
(\operatorname{div} \psi, w)+(\gamma \phi, w)=G(w), \quad w \in W_{h},
\end{gathered}
$$

which for $h$ sufficiently small has a unique solution $(\psi, \phi) \in \mathbf{V}_{h} \times W_{h}$ for any $F \in \mathbf{V}^{\prime}, G \in W^{\prime}$. (Existence follows from uniqueness. Thus it suffices to prove that if $F=0$ and $G=0$, then $(\psi, \phi)=(0,0)$. This is done in [5] by an argument entirely analogous to that of our Lemma 2.1.) We are taking in (2.8)

$$
\begin{gathered}
F(\mathbf{v})=-(p, \operatorname{div} \mathbf{v})+\left(\alpha(p) \mathbf{u}+\Gamma p-\left[\tilde{\boldsymbol{\alpha}}_{p p}(\rho) \mathbf{u}+\tilde{\boldsymbol{\beta}}_{p p}(\rho)\right](p-\rho)^{2}\right. \\
\left.+\tilde{\boldsymbol{\alpha}}_{p}(\rho)(p-\rho)(\mathbf{u}-\mu), \mathbf{v}\right), \\
G(w)=\left(\operatorname{div} \mathbf{u}+\gamma p-\tilde{c}_{p p}(\rho)(p-\rho)^{2}, w\right) .
\end{gathered}
$$

The existence of a solution $\left(\mathbf{u}_{h}, p_{h}\right) \in \mathbf{V}_{h} \times W_{h}$ of (1.11) is equivalent to that of the following problem.

Problem 2.1. The map $\Phi$ has a fixed point.

The solvability of Problem 2.1 will follow from the Brouwer fixed point theorem if we can prove that $\Phi$ maps a ball of $\mathbf{V}_{h} \times W_{h}$ into itself.

In order to do that, we shall use the following technical result.

LemMA 2.1. Let $2 \leqslant \theta<\infty$. Let $\omega \in \mathbf{V}, \mathbf{q} \in L^{2}(\Omega)^{2}$, and $r \in L^{2}(\Omega)$. If $\tau \in W_{h}$ satisfies

$$
\left\{\begin{array}{l}
(\alpha(p) \omega, \mathbf{v})-(\operatorname{div} \mathbf{v}, \tau)+(\Gamma \tau, \mathbf{v})=(\mathbf{q}, \mathbf{v}), \\
(\operatorname{div} \omega, w)+(\gamma \tau, w)=(r, w), \quad w \in \mathbf{V}_{h}
\end{array}\right.
$$

then, there exists a constant $C=C(\theta, \alpha, \Gamma, \gamma, \Omega)$ such that

$$
\|\tau\|_{0, \theta} \leqslant C\left[h^{2 / \theta}\|\boldsymbol{\omega}\|_{0}+h^{1+(2 / \theta)\left(1-\delta_{0 k}\right)}\|\operatorname{div} \boldsymbol{\omega}\|_{0}+\|\mathbf{q}\|_{0}+\|r\|_{0}\right]
$$

for $h$ sufficiently small. Also, if $\omega \in W^{0, \theta}(\operatorname{div} ; \Omega)=\left\{\mathbf{v} \in L^{\theta}(\Omega)^{2} \mid \operatorname{div} \mathbf{v} \in L^{\theta}(\Omega)\right\}$, $\mathbf{q} \in L^{\theta}(\Omega)^{2}$, and $r \in L^{\theta}(\Omega)$,

$\|\tau\|_{0, \theta} \leqslant C\left[h\|\omega\|_{0, \theta}+h^{2-\delta_{0 k}}\|\operatorname{div} \omega\|_{0, \theta}+h\|\mathbf{q}\|_{0, \theta}+h^{2-\delta_{0 k}}\|r\|_{0, \theta}+\|\mathbf{q}\|_{-1, \theta}+\|r\|_{-2, \theta}\right]$.

Here, and throughout the paper, $\delta_{i j}$ will denote the Kronecker symbol.

Proof. Let $\theta^{\prime}=\theta /(\theta-1)$ be the conjugate exponent of $\theta$. Since

$$
\|\tau\|_{0, \theta}=\sup _{\substack{\psi \in L^{\theta^{\prime}(\Omega)} \\ \psi \neq 0}} \frac{(\tau, \psi)}{\|\psi\|_{0, \theta^{\prime}}}
$$


we wish to bound $(\tau, \psi)$ for $\psi \in L^{\theta^{\prime}}(\Omega)$. Let $\phi \in W^{2, \theta^{\prime}}(\Omega)$ be the (unique) solution of $M^{*} \phi=\psi$ in $\Omega, \phi=0$ on $\partial \Omega$. Then [1],

$$
\|\phi\|_{2, \theta^{\prime}} \leqslant K\|\psi\|_{0, \theta^{\prime}} \text {. }
$$

Observe that the Sobolev imbedding theorem [17] implies that $W^{2-(2 / \theta), \theta^{\prime}}(\Omega) \subset$ $H^{1}(\Omega)$ and $W^{1-(2 / \theta), \theta^{\prime}}(\Omega) \subset L^{2}(\Omega)$; so, $W^{1, \theta^{\prime}}(\Omega) \subset H^{(2 / \theta)}(\Omega)$ with

$$
\|\lambda\|_{2 / \theta} \leqslant K\|\lambda\|_{1, \theta^{\prime}}
$$

Next, note that for any $E \in \mathscr{T}_{h}$

$$
\left.a(p) \nabla \phi \in W^{1, \theta^{\prime}}(E)^{2} \Rightarrow a(p) \nabla \phi\right|_{\partial E} \in W^{1-\left(1 / \theta^{\prime}\right), \theta^{\prime}}(\partial E)^{2},
$$

and therefore the degrees of freedom defining $\pi_{h} a(p) \nabla \phi$ on $E$ are well-defined, that is, $\pi_{h} a(p) \nabla \phi$ is well-defined.

Using (1.4), (1.10), (2.9), and integration by parts, we see that

$$
\begin{aligned}
(\tau, \psi)= & \left(\tau, M^{*} \phi\right)=(\tau,-\nabla \cdot(a(p) \nabla \phi)+a(p) \Gamma \cdot \nabla \phi+\gamma \phi) \\
= & \left(\tau,-\nabla \cdot\left(\pi_{h} a(p) \nabla \phi\right)\right)+\left(\Gamma \tau, \pi_{h} a(p) \nabla \phi\right) \\
& \quad+\left(\Gamma \tau, a(p) \nabla \phi-\pi_{h} a(p) \nabla \phi\right)+(\gamma \tau, \phi) \\
= & \left(\mathbf{q}, \pi_{h} a(p) \nabla \phi\right)-\left(\alpha(p) \omega, \pi_{h} a(p) \nabla \phi\right) \\
& \quad+\left(\Gamma \tau, a(p) \nabla \phi-\pi_{h} a(p) \nabla \phi\right)+(\gamma \tau, \phi) \\
= & (\mathbf{q}, a(p) \nabla \phi)+\left(\mathbf{q}, \pi_{h} a(p) \nabla \phi-a(p) \nabla \phi\right) \\
& +\left(\alpha(p) \omega+\Gamma \tau, a(p) \nabla \phi-\pi_{h} a(p) \nabla \phi\right)+(\operatorname{div} \omega, \phi)+(\gamma \tau, \phi) \\
= & (\mathbf{q}, a(p) \nabla \phi)+\left(\mathbf{q}, \pi_{h} a(p) \nabla \phi-a(p) \nabla \phi\right) \\
& +\left(\alpha(p) \omega+\Gamma \tau, a(p) \nabla \phi-\pi_{h} a(p) \nabla \phi\right)+\left(\operatorname{div} \omega+\gamma \tau, \phi-P_{h} \phi\right) \\
& +(r, \phi)+\left(r, P_{h} \phi-\phi\right) .
\end{aligned}
$$

First, observe that

$(\mathbf{q}, a(p) \nabla \phi) \leqslant K\|\mathbf{q}\|_{0}\|\nabla \phi\|_{0} \leqslant K\|\mathbf{q}\|_{0}\|\phi\|_{2, \theta^{\prime}}$.

Furthermore, since $\mathrm{V}(E) \supset P_{0}(E)^{2}$, an $L^{p}$-version of the Bramble-Hilbert Lemma [3] implies that (using (2.11))

$$
\left\|a(p) \nabla \phi-\pi_{h} a(p) \nabla \phi\right\|_{0} \leqslant K h^{2 / \theta}\|\nabla \phi\|_{2 / \theta} \leqslant K h^{2 / \theta}\|\nabla \phi\|_{1, \theta^{\prime}} \leqslant K h^{2 / \theta}\|\phi\|_{2, \theta^{\prime}},
$$

(a bound which cannot be obtained from (1.5) if $2 / \theta \leqslant 1 / 2$ ) and therefore

$$
\begin{aligned}
\left(\mathbf{q}-\alpha(p) \omega, \pi_{h} a(p) \nabla \phi-a(p) \nabla \phi\right) & \leqslant K\left(\|\mathbf{q}\|_{0}+\|\omega\|_{0}\right)\left\|\pi_{h} a(p) \nabla \phi-a(p) \nabla \phi\right\|_{0} \\
& \leqslant K\left(\|\mathbf{q}\|_{0}+\|\omega\|_{0}\right) h^{2 / \theta}\|\phi\|_{2, \theta^{\prime}} .
\end{aligned}
$$

Also, we see from (1.5) that

$$
\begin{aligned}
\left(\Gamma \tau, a(p) \nabla \phi-\pi_{h} a(p) \nabla \phi\right) & \leqslant K\|\tau\|_{0, \theta}\left\|a(p) \nabla \phi-\pi_{h} a(p) \nabla \phi\right\|_{0, \theta^{\prime}} \\
& \leqslant K\|\tau\|_{0, \theta} h\|\nabla \phi\|_{1, \theta^{\prime}} \leqslant K\|\tau\|_{0, \theta} h\|\phi\|_{2, \theta^{\prime}} .
\end{aligned}
$$

Finally, from (1.8) and the Sobolev imbedding theorem we see that

$$
\begin{aligned}
\left(\operatorname{div} \omega, \phi-P_{h} \phi\right) & \leqslant K\|\operatorname{div} \omega\|_{0}\left\|\phi-P_{h} \phi\right\|_{0} \\
& \leqslant K\|\operatorname{div} \omega\|_{0} h^{1+(2 / \theta)\left(1-\delta_{0 k}\right)}\|\phi\|_{1+(2 / \theta)\left(1-\delta_{0 k}\right)} \\
& \leqslant K\|\operatorname{div} \omega\|_{0} h^{1+(2 / \theta)\left(1-\delta_{0 k}\right)}\|\phi\|_{2, \theta}, \\
\left(\gamma \tau, \phi-P_{h} \phi\right) & \leqslant K\|\tau\|_{0, \theta}\left\|\phi-P_{h} \phi\right\|_{0, \theta^{\prime}} \leqslant K\|\tau\|_{0, \theta} h^{2-\delta_{0 k}}\|\phi\|_{2, \theta^{\prime}}, \\
(r, \phi)+\left(r, P_{h} \phi-\phi\right) & \leqslant K\|r\|_{0}\|\phi\|_{0} \leqslant K\|r\|_{0}\|\phi\|_{1-(2 / \theta), \theta^{\prime}} \leqslant K\|r\|_{0}\|\phi\|_{2, \theta^{\prime}} .
\end{aligned}
$$


It then follows that

$$
(\tau, \psi) \leqslant K\left[h\|\tau\|_{0, \theta}+h^{2 / \theta}\|\omega\|_{0}+h^{1+(2 / \theta)\left(1-\delta_{0 k}\right)}\|\operatorname{div} \omega\|_{0}+\|\mathbf{q}\|_{0}+\|r\|_{0}\right]\|\psi\|_{0, \theta^{\prime}},
$$

and thus, if $h$ is sufficiently small, it suffices to take $C \geqslant(1-K h)^{-1}$ in (2.10). The second bound for $\|\tau\|_{0, \theta}$ follows easily from the equation for $(\tau, \psi)$ following (2.11), (1.5), and (1.8). Q.E.D.

Let now $\mathscr{V}_{h}=\mathbf{V}_{h}$ with the stronger norm $\|\mathbf{v}\|_{\mathscr{V}_{h}}=\|\mathbf{v}\|_{0,2+\varepsilon}+\|\operatorname{div} \mathbf{v}\|_{0}$, and let $\mathscr{W}_{h}=W_{h}$ with the stronger norm $\|w\|_{\mathscr{W}_{h}}=\|w\|_{0,(4+2 \varepsilon) / \varepsilon}$.

We are now ready to demonstrate the existence of a solution of Problem 2.1.

THEOREM 2.1. For $\delta>0$ sufficiently small (dependent on $h$ ), $\Phi$ maps a ball of radius $\delta$ of $\mathscr{V}_{h} \times \mathscr{W}_{h}$ into itself.

Proof. Let $\theta=(4+2 \varepsilon) / \varepsilon$ so that $(1 / \theta)+(1 /(2+\varepsilon))=\frac{1}{2}$. Let

$$
\left\|\pi_{h} \mathbf{u}-\mu\right\|_{\mathscr{\gamma}_{h}} \leqslant \delta \quad \text { and } \quad\left\|P_{h} p-\rho\right\|_{\mathscr{W}_{h}} \leqslant \delta<1 .
$$

Interpret $\mathbf{q}$ and $r$ in (2.10) as

$$
\begin{gathered}
\mathbf{q}=\Gamma\left(P_{h} p-p\right)+\alpha(p)\left(\pi_{h} \mathbf{u}-\mathbf{u}\right) \\
+\left[\tilde{\alpha}_{p p}(\rho) \mathbf{u}+\tilde{\boldsymbol{\beta}}_{p p}(\rho)\right](p-\rho)^{2}+\tilde{\alpha}_{p}(\rho)(p-\rho)(\mathbf{u}-\mu), \\
\quad r=\gamma\left(P_{h} p-p\right)+\tilde{c}_{p p}(\rho)(p-\rho)^{2},
\end{gathered}
$$

and apply Lemma 2.1 to (2.8).

Note that, since $\varepsilon<1$ implies that $\theta>4$ and that $2+\varepsilon<4 /(2-2 \varepsilon), H^{\varepsilon}(\Omega)^{2} \subset$ $\mathrm{L}^{2+\varepsilon}(\Omega)^{2}, \quad H^{1+\varepsilon}(\Omega)^{2} \subset W^{1,2+\varepsilon}(\Omega)^{2}, \quad$ and $H^{2+\varepsilon}(\Omega)^{2} \subset W^{2,2+\varepsilon}(\Omega)^{2}$. Thus, $H^{1+(\varepsilon / 2)}(\Omega)^{2} \subset W^{1,2+\varepsilon}(\Omega)^{2}$ and $\|\lambda\|_{1,2+\varepsilon} \leqslant Q_{\varepsilon}\|\lambda\|_{1+(\varepsilon / 2)}$. Then, (1.5) and (1.8) imply the inequality

$$
\begin{aligned}
& \left\|P_{h} p-z\right\|_{0, \theta} \leqslant K\left[h^{2 / \theta}\left\|\pi_{h} \mathbf{u}-\mathbf{y}\right\|_{0}+h^{1+(2 / \theta)\left(1-\delta_{0 k}\right)}\left\|\operatorname{div}\left(\pi_{h} \mathbf{u}-\mathbf{y}\right)\right\|_{0}\right. \\
& \left.+\|\mathbf{q}\|_{0}+\|r\|_{0}\right] \\
& \leqslant K\left[h^{2 / \theta}\left\|\pi_{h} \mathbf{u}-\mathbf{y}\right\|_{0}+h^{1+(2 / \theta)\left(1-\delta_{0 k}\right)}\left\|\operatorname{div}\left(\pi_{h} \mathbf{u}-\mathbf{y}\right)\right\|_{0}\right. \\
& \left.+h\|p\|_{1}+h\|\mathbf{u}\|_{1}+\|p-\rho\|_{0,4}^{2}+\|p-\rho\|_{0, \theta}\|\mathbf{u}-\mu\|_{0,2+\varepsilon}\right] \\
& \leqslant K\left[h^{2 / \theta}\left\|\pi_{h} \mathbf{u}-\mathbf{y}\right\|_{0}+h^{1+(2 / \theta)\left(1-\delta_{0 k}\right)}\left\|\operatorname{div}\left(\pi_{h} \mathbf{u}-\mathbf{y}\right)\right\|_{0}\right. \\
& +h\|p\|_{2}+\|p-\rho\|_{0, \theta}^{2}+\left(\left\|p-P_{h} p\right\|_{0, \theta}+\left\|P_{h} p-\rho\right\|_{0, \theta}\right) \\
& \left.\left(\left\|\mathbf{u}-\pi_{h} \mathbf{u}\right\|_{0,2+\varepsilon}+\left\|\pi_{h} \mathbf{u}-\mu\right\|_{0,2+\varepsilon}\right)\right] \\
& \leqslant K\left[h^{2 / \theta}\left\|\pi_{h} \mathbf{u}-\mathbf{y}\right\|_{0}+h^{1+(2 / \theta)\left(1-\delta_{0 k}\right)}\left\|\operatorname{div}\left(\pi_{h} \mathbf{u}-\mathbf{y}\right)\right\|_{0}\right. \\
& \left.+h\|p\|_{2}+\delta^{2}+\left(h\|p\|_{1, \theta}+\delta\right)\left(h\|\mathbf{u}\|_{1,2+\varepsilon}+\delta\right)\right] \\
& \leqslant K\left[h^{2 / \theta}\left\|\pi_{k} \mathbf{u}-\mathbf{y}\right\|_{0}+h^{1+(2 / \theta)-\delta_{0 k}}\left\|\operatorname{div}\left(\pi_{h} \mathbf{u}-\mathbf{y}\right)\right\|_{0}+\left(h+\delta^{2}\right)\|p\|_{2+\varepsilon}^{2}\right] \text {. }
\end{aligned}
$$


If we now take the last term on the left side of each equation in (2.8) over to the right side, the left side becomes exactly the mixed method equations for the operator $-\nabla \cdot(a(p) \nabla)$. It follows from [2] that then

$$
\begin{aligned}
\left\|\pi_{h} \mathbf{u}-\mathbf{y}\right\|_{\mathbf{v}} & \leqslant K\left[\left\|P_{h} p-z\right\|_{0}+\|\mathbf{q}\|_{0}+\|r\|_{0}\right] \\
& \leqslant K\left[\left\|P_{h} p-z\right\|_{0, \theta}+\left(h+\delta^{2}\right)\|p\|_{2+\varepsilon}^{2}\right] .
\end{aligned}
$$

If we now substitute (2.13) into (1.12), we see that, for $h$ sufficiently small,

$$
\left\|P_{h} p-z\right\|_{0, \theta} \leqslant K_{1}\left[h+\delta^{2}\right]
$$

with $K_{1}$ depending on $\|p\|_{2+\varepsilon}^{2}$ linearly. Putting (2.14) back into (2.13), we obtain (with $K_{2}=K\left(K_{1}+\|p\|_{2+\varepsilon}^{2}\right)$ )

$$
\begin{gathered}
\left\|\operatorname{div}\left(\pi_{h} \mathbf{u}-\mathbf{y}\right)\right\|_{0} \leqslant K_{2}\left[h+\delta^{2}\right], \\
\left\|\pi_{h} \mathbf{u}-\mathbf{y}\right\|_{0} \leqslant K_{2}\left[h+\delta^{2}\right] .
\end{gathered}
$$

It follows from (2.16), using the quasi-regularity of $\mathscr{T}_{h}$, that

$$
\begin{aligned}
\left\|\pi_{h} \mathbf{u}-\mathbf{y}\right\|_{0,2+\varepsilon} & \leqslant K_{\varepsilon} h^{(2 /(2+\varepsilon))-1}\left\|\pi_{h} \mathbf{u}-\mathbf{y}\right\|_{0} \\
& \leqslant K_{\varepsilon} h^{-(\varepsilon /(2+\varepsilon))} K_{2}\left(h+\delta^{2}\right) \leqslant K_{3}\left[h^{2 /(2+\varepsilon)}+h^{-(\varepsilon /(2+\varepsilon))} \delta^{2}\right] .
\end{aligned}
$$

We now see that (2.15) and (2.17) imply that

$$
\left\|\pi_{h} \mathbf{u}-\mathbf{y}\right\|_{\gamma_{h}} \leqslant 2 K_{3}\left[h^{2 /(2+\varepsilon)}+h^{-(\varepsilon /(2+\varepsilon))} \delta^{2}\right] .
$$

Now let $h<\left(4 K_{3}\right)^{-(4+2 \varepsilon) /(2-\varepsilon)}$ and take $\delta=4 K_{3} h^{2 /(2+\varepsilon)}$. Observe that in order that $2 K_{3} h^{2 /(2+\varepsilon)} \leqslant \delta / 2$ and $2 K_{3} h^{-(\varepsilon / 2+\varepsilon))} \delta^{2} \leqslant \delta / 2$, we must have $\delta \in\left[4 K_{3} h^{2 /(2+\varepsilon)}\right.$, $\left.\left(4 K_{3}\right)^{-1} h^{\varepsilon /(2+\varepsilon)}\right] \neq \varnothing$, which is satisfied for $h$ and $\delta$ as chosen.

The theorem is now proved, since (2.14) and (2.18) imply that $\left\|P_{h} p-z\right\|_{\mathscr{W}_{h}} \leqslant \delta$ and $\left\|\pi_{h} \mathbf{u}-\mathbf{y}\right\|_{\mathscr{r}_{h}} \leqslant \delta$; that is, $\Phi$ maps the balls of radius $\delta=O\left(h^{2 /(2+\varepsilon)}\right)$, centered at $\left(\pi_{h} \mathbf{u}, P_{h} p\right)$ into itself. Q.E.D.

3. $L^{2}$-Error Estimates. Note that Theorem 2.1 in fact shows that as $h \rightarrow 0$ we obtain a sequence $\left\{\left(\mathbf{u}_{h}, p_{h}\right)\right\}_{h \downarrow 0}$ which converges to $(\mathbf{u}, p)$ in $\mathbf{V} \cap L^{2+\varepsilon}(\Omega)^{2} \times L^{\theta}(\Omega)$ and furthermore, that there is a constant $C=4 K_{3}+Q Q_{\varepsilon}\|\mathbf{u}\|_{1+\varepsilon}$ such that

$$
\max \left\{\left\|\mathbf{u}-\mathbf{u}_{h}\right\|_{0,2+\varepsilon} ;\left\|p-p_{h}\right\|_{0, \theta}\right\} \leqslant C h^{2 /(2+\varepsilon)} \text {, }
$$

since

$$
\begin{aligned}
\left\|\mathbf{u}-\mathbf{u}_{h}\right\|_{0,2+\varepsilon} & \leqslant\left\|\mathbf{u}-\pi_{h} \mathbf{u}\right\|_{0,2+\varepsilon}+\left\|\pi_{h} \mathbf{u}-\mathbf{u}_{h}\right\|_{0,2+\varepsilon} \\
& \leqslant Q h\|\mathbf{u}\|_{1,2+\varepsilon}+\delta \leqslant Q h Q_{\varepsilon}\|\mathbf{u}\|_{1+\varepsilon}+4 K_{3} h^{2 /(2+\varepsilon)}
\end{aligned}
$$

and

$$
\begin{aligned}
\left\|p-p_{h}\right\|_{0, \theta} & \leqslant\left\|p-P_{h} p\right\|_{0, \theta}+\left\|P_{h} p-p_{h}\right\|_{0, \theta} \\
& \leqslant Q h\|p\|_{1, \theta}+\delta \leqslant Q h Q_{\varepsilon}\|p\|_{2}+4 K_{3} h^{2 /(2+\varepsilon)} .
\end{aligned}
$$

Let us now rewrite (2.4) as

$$
\text { (a) } \quad \begin{aligned}
\left(\alpha(p)\left[\mathbf{u}-\mathbf{u}_{h}\right], \mathbf{v}\right)-\left(\operatorname{div} \mathbf{v}, p-p_{h}\right) \\
+\left(\left[\tilde{\alpha}_{p}\left(p_{h}\right) \mathbf{u}_{h}+\tilde{\boldsymbol{\beta}}_{p}\left(p_{h}\right)\right]\left(p-p_{h}\right), \mathbf{v}\right)=0, \quad \mathbf{v} \in \mathbf{V}_{h}
\end{aligned}
$$

(b) $\left(\operatorname{div}\left[\mathbf{u}-\mathbf{u}_{h}\right], w\right)+\left(\tilde{c}_{p}\left(p_{h}\right)\left[p-p_{h}\right], w\right)=0, \quad w \in W_{h}$,

where $\tilde{\beta}_{p}\left(p_{h}\right)$ and $\tilde{c}_{p}\left(p_{h}\right)$ are bounded functions in $\bar{\Omega}$ defined in (2.2) and (2.3). 
Observe that (3.2) corresponds to the mixed method for the operator $N: H^{2}(\Omega) \cap$ $H_{0}^{1}(\Omega) \rightarrow L^{2}(\Omega)$ given by

$$
N w=-\nabla \cdot\left(a(p) \nabla w+a(p)\left[\tilde{\alpha}_{p}\left(p_{h}\right) \mathbf{u}_{h}+\tilde{\beta}_{p}\left(p_{h}\right)\right] w\right)+\tilde{c}_{p}\left(p_{h}\right) w .
$$

Its formal adjoint $N^{*}: H^{2}(\Omega) \cap H_{0}^{1}(\Omega) \rightarrow L^{2}(\Omega)$ is

$$
N^{*} \chi=-\nabla \cdot(a(p) \nabla \chi)+a(p)\left[\tilde{\alpha}_{p}\left(p_{h}\right) \mathbf{u}_{h}+\tilde{\beta}_{p}\left(p_{h}\right)\right] \cdot \nabla \chi+\tilde{c}_{p}\left(p_{h}\right) \chi .
$$

Before we turn to the rate of convergence of $\left(\mathbf{u}_{h}, p_{h}\right)$ to $(\mathbf{u}, p)$, we need the following technical result.

Lemma 3.1. There exists an $h_{0}>0$ such that, if $h<h_{0}, N^{*}$ has a bounded inverse mapping $L^{2}(\Omega)$ onto $H^{2}(\Omega) \cap H_{0}^{1}(\Omega)$.

Proof. Since $M^{*-1}: L^{2}(\Omega) \rightarrow H^{2}(\Omega) \cap H_{0}^{1}(\Omega)$ is bounded and $N^{*-1}=$ $\left(M^{*-1} N^{*}\right)^{-1} M^{*-1}$, it suffices to show that $M^{*-1} N^{*}$ has a bounded inverse on $H^{2}(\Omega) \cap H_{0}^{1}(\Omega)$. For a linear differential operator $D: X \rightarrow Y$, let $\|D\|$ be its norm as a linear functional; e.g.

$$
\left\|M^{*}\right\|\|=\| M^{*} \|_{\mathscr{L}\left(H^{2}(\Omega) \cap H_{0}^{1}(\Omega) ; L^{2}(\Omega)\right)} .
$$

Then, all that is needed is to prove that $\left\|\left|M^{*-1}\left(M^{*}-N^{*}\right) \|\right|\right.$ is less than one, since this will imply that $I-M^{*-1}\left(M^{*}-N^{*}\right)=M^{*-1} N^{*}$ has a bounded inverse. Thus, it is sufficient to show that $\left\|M^{*}-N^{*}\right\| \mid$ is smaller than $\left(\left\|\left|M^{*-1} \|\right|\right)^{-1}\right.$.

We have, by (2.7) and (3.3),

$$
\begin{aligned}
\left(M^{*}-N^{*}\right) \chi= & a(p)\left[\alpha_{p}(p) \mathbf{u}-\tilde{\alpha}_{p}\left(p_{h}\right) \mathbf{u}_{h}+\boldsymbol{\beta}_{p}(p)-\tilde{\boldsymbol{\beta}}_{p}\left(p_{h}\right)\right] \cdot \nabla \chi \\
& +\left(c_{p}(p)-\tilde{c}_{p}\left(p_{h}\right)\right) \chi \\
= & a(p)\left[\left(\alpha_{p}(p)-\tilde{\boldsymbol{\alpha}}_{p}\left(p_{h}\right)\right) \mathbf{u}+\tilde{\boldsymbol{\alpha}}_{p}\left(p_{h}\right)\left(\mathbf{u}-\mathbf{u}_{h}\right)\right. \\
& \left.+\left(\beta_{p}(p)-\tilde{\boldsymbol{\beta}}_{p}\left(p_{h}\right)\right)\right] \cdot \nabla \chi \\
& +\left(c_{p}(p)-\tilde{c}\left(p_{h}\right)\right) \chi .
\end{aligned}
$$

Observe that $\xi=p-p_{h}$,

$$
\begin{aligned}
\alpha_{p}(p)-\tilde{\alpha}_{p}\left(p_{h}\right) & =\int_{0}^{1}\left[\alpha_{p}(p)-\alpha_{p}\left(p_{h}+t \xi\right)\right] d t \\
& =\xi \int_{0}^{1}(1-t) \int_{0}^{1} \alpha_{p p}\left(p_{h}+t \xi+s(1-t) \xi\right) d s d t \\
& =\bar{\alpha}_{p p} \xi
\end{aligned}
$$

where $\bar{\alpha}_{p p}$ is a bounded function. Similarly, we obtain

$$
\boldsymbol{\beta}_{p}(p)-\tilde{\boldsymbol{\beta}}_{p}\left(p_{h}\right)=\overline{\boldsymbol{\beta}}_{p p} \xi, \quad c_{p}(p)-\tilde{c}_{p}\left(p_{h}\right)=\bar{c}_{p p} \xi
$$

where $\bar{\beta}_{p p}$ and $\bar{c}_{p p}$ are bounded functions. Substituting (3.5) and (3.6) into (3.4), we see that

$$
\begin{aligned}
\left(M^{*}-N^{*}\right) \chi= & a(p)\left\{\left[\overline{\boldsymbol{\alpha}}_{p p} \mathbf{u}+\overline{\boldsymbol{\beta}}_{p p}\right]\left(p-p_{h}\right)+\tilde{\boldsymbol{\alpha}}_{p}\left(p_{h}\right)\left(\mathbf{u}-\mathbf{u}_{h}\right)\right\} \cdot \nabla \chi \\
& +\bar{c}_{p p}\left(p-p_{h}\right) \chi
\end{aligned}
$$


and thus, using (3.1),

$$
\begin{aligned}
\left\|\left(M^{*}-N^{*}\right) \chi\right\|_{0} \leqslant & K_{4}\left[\|\mathbf{u}\|_{0, \infty}\left\|p-p_{h}\right\|_{0,(4+2 \varepsilon) / \varepsilon}\|\nabla \chi\|_{0,2+\varepsilon}\right. \\
& \left.+\left\|\mathbf{u}-\mathbf{u}_{h}\right\|_{0,2+\varepsilon}\|\nabla \chi\|_{0,(4+2 \varepsilon) / \varepsilon}+\left\|p-p_{h}\right\|_{0}\|\chi\|_{0, \infty}\right] \\
\leqslant & K_{5}\|p\|_{2+\varepsilon}^{3}\left[h^{2 /(2+\varepsilon)}\|\nabla \chi\|_{1}+h^{2 /(2+\varepsilon)}\|\chi\|_{1+\varepsilon}\right] \\
\leqslant & K_{6} h^{2 /(2+\varepsilon)}\|\chi\|_{2},
\end{aligned}
$$

since $H^{1}(\Omega)^{2} \subset L^{r}(\Omega)^{2}$ for any finite $r$ and $H^{1+\varepsilon}(\Omega) \subset L^{\infty}(\Omega)$. Now, take $h_{0}$ small enough that $K_{6} h_{0}^{2 /(2+\varepsilon)} \leqslant\left(\|\left|M^{*-1}\right| \mid\right)^{-1}$. Q.E.D.

We can now obtain a rate of convergence of $\left(\mathbf{u}_{h}, p_{h}\right)$ to $(\mathbf{u}, p)$ as $h \rightarrow 0$.

THEOREM 3.1. There is a positive constant $C$ independent of $h$, depending on $\|p\|_{2+\varepsilon}$ quadratically such that

$$
\left\|p-p_{h}\right\|_{0} \leqslant C\left\{\begin{array}{l}
h\|p\|_{2}, \quad \text { if } k=0, \\
h^{s}\|p\|_{s}, \quad 2 \leqslant s \leqslant k+1, \text { if } p \in H^{s} \text { and } k>0,
\end{array}\right.
$$

(ii) $\quad\left\|\mathbf{u}-\mathbf{u}_{h}\right\|_{0} \leqslant C h^{s}\|p\|_{s+1}, \quad 1 \leqslant s \leqslant k+1$, if $p \in H^{s+1}(\Omega)$,

(iii) $\quad\left\|\operatorname{div}\left(\mathbf{u}-\mathbf{u}_{h}\right)\right\|_{0} \leqslant C h^{s}\|p\|_{s+2}, \quad 0 \leqslant s \leqslant k+1$, if $p \in H^{s+2}(\Omega)$.

Proof. Let $\zeta=\mathbf{u}-\mathbf{u}_{h}, \xi=p-p_{h}, \boldsymbol{\sigma}=\pi_{h} \mathbf{u}-\mathbf{u}_{h}$, and $\tau=P_{h} p-p_{h}$. Rewrite (3.2) in the form

$$
\left\{\begin{aligned}
&(\alpha(p) \zeta, v)-(\operatorname{div} \mathbf{v}, \tau)+\left(\left[\tilde{\alpha}_{p}\left(p_{h}\right) \mathbf{u}_{h}+\tilde{\boldsymbol{\beta}}_{p}\left(p_{h}\right)\right] \tau, \mathbf{v}\right) \\
&=\left(\left[\tilde{\alpha}_{p}\left(p_{h}\right) \mathbf{u}_{h}+\tilde{\boldsymbol{\beta}}_{p}\left(p_{h}\right)\right]\left[P_{h} p-p\right], \mathbf{v}\right), \mathbf{v} \in \mathbf{V}_{h}, \\
&(\operatorname{div} \zeta, w)+\left(\tilde{c}_{p}\left(p_{h}\right) \tau, w\right)=\left(\tilde{c}_{p}\left(p_{h}\right)\left[P_{h} p-p\right], w\right), \quad w \in W_{h} .
\end{aligned}\right.
$$

It follows from Lemma 3.1 of [5] and our Lemma 3.1 that

$$
\begin{aligned}
\|\tau\|_{0} \leqslant K\left[h\|\zeta\|_{0}+h^{2-\delta_{0 k}}\|\operatorname{div} \zeta\|_{0}+\|\left[\tilde{\alpha}_{p}\left(p_{h}\right) \mathbf{u}_{h}+\right.\right. & \left.\tilde{\beta}_{p}\left(p_{h}\right)\right]\left(P_{h} p-p\right) \|_{0} \\
& \left.+\left\|\tilde{c}_{p}\left(p_{h}\right)\left(P_{h} p-p\right)\right\|_{0}\right] .
\end{aligned}
$$

If $p \in H^{s}(\Omega)$, then $p \in H^{s-(2 /(2+\varepsilon)), \theta}(\Omega)$ and $\|p\|_{s-(2 /(2+\varepsilon)), \theta} \leqslant K\|p\|_{s}$. Thus, using (1.8) and (3.1), the penultimate term in (3.7) can be bounded by

$$
\begin{aligned}
\|\left[\tilde{\boldsymbol{\alpha}}_{p}\left(p_{h}\right) \mathbf{u}_{h}+\right. & \left.\tilde{\boldsymbol{\beta}}_{h}\left(p_{h}\right)\right]\left(P_{h} p-p\right) \|_{0} \\
& \leqslant K\left[\|\mathbf{u}\|_{0, \infty}\left\|P_{h} p-p\right\|_{0}+\left\|\mathbf{u}-\mathbf{u}_{h}\right\|_{0,2+\varepsilon}\left\|P_{h} p-p\right\|_{0,(4+2 \varepsilon) / \varepsilon}\right] \\
& \leqslant K\|p\|_{2+\varepsilon}^{2}\left[h^{s}\|p\|_{s}+h^{2 /(2+\varepsilon)} h^{s-(2 /(2+\varepsilon))}\|p\|_{s-(2 /(2+\varepsilon)), \theta}\right] \\
& \leqslant K\|p\|_{2+\varepsilon}^{2} h^{s}\|p\|_{s} .
\end{aligned}
$$

We now derive a preliminary bound for $\|\xi\|_{0}$. Substituting (1.8) and (3.8) into (3.7) gives the bound

$$
\|\tau\|_{0} \leqslant K\left[h\|\zeta\|_{0}+h^{2-\delta_{0 k}}\|\operatorname{div} \zeta\|_{0}+h^{s}\|p\|_{s}\|p\|_{2+\varepsilon}^{2}\right],
$$

which in turn implies, using again (1.8), that

$$
\begin{aligned}
\|\xi\|_{0} & =\left\|p-p_{h}\right\|_{0} \leqslant\left\|p-P_{h} p\right\|_{0}+\|\tau\|_{0} \\
& \leqslant K\left[\|p\|_{2+\varepsilon}^{2} h^{s}\|p\|_{s}+h^{2-\delta_{0 k}}\|\operatorname{div} \zeta\|_{0}+h\|\zeta\|_{0}\right] .
\end{aligned}
$$


The quasi-regularity of $\mathscr{T}_{h}$ implies that

$$
\|\boldsymbol{\sigma}\|_{0, \infty} \leqslant K h^{-2 /(2+\varepsilon)}\|\boldsymbol{\sigma}\|_{0,2+\varepsilon} .
$$

The Sobolev imbedding theorem implies that

$$
H^{1+\varepsilon}(\Omega)^{2} \subset W^{(\varepsilon / 2), \infty}(\Omega)^{2} .
$$

Using (1.5) with $q=\infty$ and $s=\varepsilon / 2$, we obtain from (3.1), (3.10), and (3.11) the inequality

$$
\begin{aligned}
\left\|\mathbf{u}_{h}\right\|_{0, \infty} & \leqslant\|\mathbf{u}\|_{0, \infty}+\left\|\pi_{h} \mathbf{u}-\mathbf{u}\right\|_{0, \infty}+\|\boldsymbol{\sigma}\|_{0, \infty} \\
& \leqslant K\left[\|p\|_{2+\varepsilon}+h^{\varepsilon / 2}\|\mathbf{u}\|_{\varepsilon / 2, \infty}+h^{-2 /(2+\varepsilon)}\|\boldsymbol{\sigma}\|_{0,2+\varepsilon}\right] \\
& \leqslant K\left[\|p\|_{2+\varepsilon}+h^{-2 /(2+\varepsilon)}\left(\left\|\pi_{h} \mathbf{u}-\mathbf{u}\right\|_{0,2+\varepsilon}+\left\|\mathbf{u}-\mathbf{u}_{h}\right\|_{0,2+\varepsilon}\right)\right] \\
& \leqslant K\left[\|p\|_{2+\varepsilon}+h^{-2 /(2+\varepsilon)}\left(Q h\|\mathbf{u}\|_{1+\varepsilon}+C h^{2 /(2+\varepsilon)}\right)\right] \leqslant K\left(\|p\|_{2+\varepsilon}^{2}+1\right) .
\end{aligned}
$$

If we now rewrite (2.4) as

$$
\left\{\begin{array}{l}
(\alpha(p) \boldsymbol{\sigma}, \mathbf{v})-(\operatorname{div} \mathbf{v}, \tau)=\left(\alpha(p)\left[\pi_{h} \mathbf{u}-\mathbf{u}\right]-\left[\tilde{\alpha}_{p}\left(p_{h}\right) \mathbf{u}_{h}+\tilde{\boldsymbol{\beta}}_{h}\left(p_{h}\right)\right] \xi, \mathbf{v}\right), \\
\mathbf{v} \in \mathbf{V}_{h}, \\
(\operatorname{div} \boldsymbol{\sigma}, w)=\left(-\tilde{c}_{p}\left(p_{h}\right) \xi, w\right), \quad w \in W_{k},
\end{array}\right.
$$

we see using [2] (just as we did to obtain (2.13)), (1.5), and (3.12), that for $\frac{1}{2}<s \leqslant k+1$ and $p \in H^{s+1}(\Omega)$

$$
\|\boldsymbol{\sigma}\|_{\mathbf{v}} \leqslant K\|p\|_{2+\varepsilon}^{2}\left[\left\|\pi_{h} \mathbf{u}-\mathbf{u}\right\|_{0}+\|\xi\|_{0}\right] \leqslant K\|p\|_{2+\varepsilon}^{2}\left[\|\xi\|_{0}+h^{s}\|p\|_{s+1}\right]
$$

From (3.13) we now obtain by (1.5) and (1.6) the bounds

$$
\begin{aligned}
\|\boldsymbol{\zeta}\|_{0} & \leqslant\left\|\mathbf{u}-\pi_{h} \mathbf{u}\right\|_{0}+\|\boldsymbol{\sigma}\|_{0} \\
& \leqslant K\left(\|p\|_{2+\varepsilon}^{2}+1\right)\left[\|\xi\|_{0}+h^{s}\|p\|_{s+1}\right], \quad 1 \leqslant s \leqslant k+1, \\
\|\operatorname{div} \zeta\|_{0} & \leqslant\left\|\operatorname{div}\left(\mathbf{u}-\pi_{h} \mathbf{u}\right)\right\|_{0}+\|\operatorname{div} \boldsymbol{\sigma}\|_{0} \\
& \leqslant K\left(\|p\|_{2+\varepsilon}^{2}+1\right)\left[\|\xi\|_{0}+h^{s}\|p\|_{s+2}\right], \quad 0 \leqslant s \leqslant k+1
\end{aligned}
$$

which, when substituted into (3.9), yield the estimate

$$
\begin{aligned}
\|\xi\|_{0} & \leqslant K\|p\|_{2+\varepsilon}^{2}\left[h\|\xi\|_{0}+h^{s-\delta_{0 k}}\|p\|_{s}+h^{s}\|p\|_{s}\right] \\
& \leqslant K\left(\|p\|_{2+\varepsilon}^{2}+1\right)\left[h\|\xi\|_{0}+h^{s-\delta_{0 k}}\|p\|_{s}\right], \quad 2 \leqslant s \leqslant k+1+\delta_{0 k}
\end{aligned}
$$

But (3.16) now implies (i) holds if $h$ is small enough. Applying (i) to (3.14) and (3.15) shows that (ii) and (iii) also hold. Q.E.D.

Observe that Theorem 3.1 shows that $\left\{\left(\mathbf{u}_{h}, p_{h}\right)\right\}_{h \downarrow 0}$ converges in $\mathbf{V} \times W$ to $(\mathbf{u}, p)$ both at an optimal rate (for any $h$ ) and with minimal smoothness requirements on the solution of (1.1) (if $k \geqslant 1$ ). 
4. A Uniqueness Result for the Discrete Problem. We shall now prove a uniqueness result.

THEOREM 4.1. If $f \in H^{2+\varepsilon+(1-\varepsilon) \delta_{0 k}}(\Omega)$ and $h$ is sufficiently small, then for any $K>0$ there is a unique solution of (1.11) in the intersection of the balls

$$
\begin{aligned}
\left\{\left\|\mathbf{u}-\mathbf{u}_{h}\right\|_{\gamma_{h}}+\left\|p-p_{h}\right\|_{\mathscr{W}_{h}} \leqslant\left(K_{4}\|\mathbf{u}\|_{0, \infty}\left\|M^{*-1}\right\|_{\mathscr{L}\left(L^{2}(\Omega) ; H^{2}(\Omega) \cap H_{0}^{1}(\Omega)\right)}\right)^{-1}\right\} & \\
& \cap\left\{\left\|\mathbf{u}-\mathbf{u}_{h}\right\|_{0, \infty}+\left\|p-p_{h}\right\|_{0, \infty} \leqslant K\right\}=B,
\end{aligned}
$$

where $K_{4}$ is the constant of Lemma 3.1 appearing after (3.6).

Proof. First note that Theorem 3.1 in fact shows that any solution $\left(\mathbf{u}_{h}, p_{h}\right) \in \mathbf{V}_{h}$ $\times W_{h}$ of (1.11) lying in $B$ will verify the bounds (i), (ii), and (iii) of Theorem 3.1. Assume now that, for $i=1$ and $2,\left(\mathbf{u}_{h}^{(i)}, p_{h}^{(i)}\right) \in \mathbf{V}_{h} \times W_{h}$ is a solution which satisfies the above hypotheses. Let $\boldsymbol{\zeta}_{i}=\mathbf{u}-\mathbf{u}_{h}^{(i)}, \xi_{i}=p-p_{h}^{(i)}, \mathbf{U}=\mathbf{u}_{h}^{(1)}-\mathbf{u}_{h}^{(2)}, P=p_{h}^{(1)}-p_{h}^{(2)}$, and $\boldsymbol{\sigma}_{i}=\pi_{h} \mathbf{u}-\mathbf{u}_{h}^{(i)}$. Theorem 5.1(b) below will then imply that

$$
\left\|\xi_{i}\right\|_{0, \infty} \leqslant Q h^{\varepsilon / 2}\|p\|_{2+\varepsilon+(1-\varepsilon) \delta_{0 k}}, \quad i=1,2 .
$$

Also, Theorem 3.1(b) implies

$$
\left\|\zeta_{i}\right\|_{0} \leqslant Q h\left\|_{p}\right\|_{2}, \quad i=1,2 .
$$

It follows from (1.5), (3.10), and (4.2) that

$$
\begin{aligned}
\left\|\boldsymbol{\zeta}_{i}\right\|_{0, \infty} & \leqslant\left\|\mathbf{u}-\pi_{h} \mathbf{u}\right\|_{0, \infty}+\left\|\boldsymbol{\sigma}_{i}\right\|_{0, \infty} \leqslant K\left[h^{\varepsilon / 2}\|\mathbf{u}\|_{\varepsilon / 2, \infty}+h^{-1}\left\|\boldsymbol{\sigma}_{i}\right\|_{0}\right] \\
& \leqslant K\left[h^{\varepsilon / 2}\|\mathbf{u}\|_{1+\varepsilon}+h^{-1}\left(\left\|\pi_{h} \mathbf{u}-\mathbf{u}\right\|_{0}+\left\|\boldsymbol{\zeta}_{i}\right\|_{0}\right)\right] \\
& \leqslant K\left[h^{\varepsilon / 2}\|p\|_{2+\varepsilon}+h^{-1} h\|p\|_{2}\right] \leqslant K(p), \quad i=1,2,
\end{aligned}
$$

where $K(p)$ depends on $\|p\|_{2+\varepsilon}$ quadratically.

It follows from (1.3) and (1.11) that

$$
\begin{aligned}
&(\alpha(p) \mathbf{U}, \mathbf{v})-(\operatorname{div} \mathbf{v}, P)=\left(\left[\alpha\left(p_{h}^{(2)}\right)-\alpha\left(p_{h}^{(1)}\right)\right] \mathbf{u}_{h}^{(2)}, \mathbf{v}\right) \\
&+\left(\left[\alpha(p)-\alpha\left(p_{h}^{(1)}\right)\right] \mathbf{U}+\boldsymbol{\beta}\left(p_{h}^{(2)}\right)-\boldsymbol{\beta}\left(p_{h}^{(1)}\right), \mathbf{v}\right), \\
& \mathbf{v} \in \mathbf{V}_{h},
\end{aligned}
$$

$$
(\operatorname{div} \mathbf{U}, w)=\left(c\left(p_{h}^{(2)}\right)-c\left(p_{h}^{(1)}\right), w\right), \quad w \in W_{h}
$$

Let

$$
\begin{array}{ll}
\alpha\left(p_{h}^{(1)}\right)-\alpha\left(p_{h}^{(2)}\right)=\bar{\alpha}_{p}(\bar{P}) P, & \alpha(p)-\alpha\left(p_{h}^{(1)}\right)=\bar{\alpha}_{p}\left(\xi_{1}\right) \xi_{1}, \\
\beta\left(p_{h}^{(1)}\right)-\beta\left(p_{h}^{(2)}\right)=\bar{\beta}_{p}(\bar{P}) P, & c\left(p_{h}^{(1)}\right)-c\left(p_{h}^{(2)}\right)=\bar{c}_{p}(\bar{P}) P,
\end{array}
$$

where $\bar{\alpha}_{p}(\bar{P}), \bar{\alpha}_{p}\left(\xi_{1}\right), \bar{\beta}_{p}(\bar{P})$, and $\bar{c}_{p}(\bar{P})$ are bounded functions in $\bar{\Omega}$, where $\bar{P}$ is some convex combination of $p_{h}^{(1)}$ and $p_{h}^{(2)}$. Then

$$
\left\{\begin{aligned}
(\alpha(p) \mathbf{U}, \mathbf{v})-(\operatorname{div} \mathbf{v}, P) & =-\left(\left[\bar{\alpha}_{p}(\bar{P}) \mathbf{u}_{h}^{(2)}+\bar{\beta}_{p}(\bar{P})\right] P, \mathbf{v}\right) \\
+\left(\bar{\alpha}_{p}\left(\xi_{1}\right) \xi_{1} \mathbf{U}, \mathbf{v}\right), \quad \mathbf{v} \in \mathbf{V}_{h}, & \\
(\operatorname{div} \mathbf{U}, w) & =-\left(\bar{c}_{p}(\bar{P}) P, w\right), \quad w \in W_{h} .
\end{aligned}\right.
$$

It follows from [2], (4.1), and (4.3) that

(4.5) $\|\mathbf{U}\|_{0} \leqslant K(p)\left[\|P\|_{0}+h\|\mathbf{U}\|_{0}\right], \quad\|\operatorname{div} \mathbf{U}\|_{0} \leqslant K(p)\left[\|P\|_{0}+h\|\mathbf{U}\|_{0}\right]$.

For $h$ sufficiently small, (4.5) implies

$$
\|\mathbf{U}\|_{0} \leqslant K(p)\|P\|_{0}
$$




$$
\|\operatorname{div} \mathbf{U}\|_{0} \leqslant K(p)\|P\|_{0} .
$$

Rewrite (4.4) in the form

$$
\begin{aligned}
(\alpha(p) \mathbf{U}, \mathbf{v})-(\operatorname{div} \mathbf{v}, P)+( & {\left.\left[\overline{\boldsymbol{\alpha}}_{p}(\bar{P}) \mathbf{u}_{h}^{(2)}+\overline{\boldsymbol{\beta}}_{p}(\bar{P})\right] P, \mathbf{v}\right)=\left(\bar{\alpha}_{p}\left(\xi_{1}\right) \xi_{1} \mathbf{U}, \mathbf{v}\right), \quad \mathbf{v} \in \mathbf{V}_{h}, } \\
(\operatorname{div} \mathbf{U}, w)+\left(\bar{c}_{p}(\bar{P}) P, w\right) & =0, \quad w \in W_{h} .
\end{aligned}
$$

Then, it follows from [4], an obvious variation of Lemma 3.1, and (4.1) that

$$
\|P\|_{0} \leqslant K(p)\left[h\|\mathbf{U}\|_{0}+h\|\operatorname{div} \mathbf{U}\|_{0}\right] .
$$

If we now substitute (4.6) and (4.7) into (4.8), we have $\|P\|_{0} \leqslant K h\|P\|_{0}$, which implies that $P=0$ for $h$ sufficiently small. Then (4.6) implies that $U=0$. Q.E.D.

5. $L^{q}$-Error Estimates $(2 \leqslant q \leqslant \infty)$. We shall first obtain a negative norm estimate for $\tau$.

Lemma 5.1. There exists a positive constant $C \leqslant K\left(\|p\|_{2+\varepsilon}^{4}+1\right)$, independent of $h$, such that, if $\partial \Omega$ and the coefficients $a, \mathbf{b}$, and $c$ of (1.1a) are sufficiently smooth, then for $0 \leqslant s \leqslant k$,

$$
\|\tau\|_{-s} \leqslant C\left[h^{r_{1}+s+1}\|p\|_{r_{1}+1+(s-k+1)^{+}}+h^{r_{2}+l-\varepsilon}\|p\|_{r_{2}+1}\|p\|_{l+\delta_{0 k}}\right],
$$

$1 \leqslant r_{1}, r_{2} \leqslant k+1,2-\delta_{0 k} \leqslant l \leqslant k+1$, where $p$ is the solution of (1.1).

Proof. Since

$$
\|\tau\|_{-s}=\sup _{\substack{\psi \in H^{s}(\Omega) \\ \psi \neq 0}} \frac{(\tau, \psi)}{\|\psi\|_{s}},
$$

we wish to bound $(\tau, \psi)$ for $\psi \in H^{s}(\Omega)$. Let $\phi \in H^{s+2}(\Omega)$ be the (unique) solution of $M^{*} \phi=\psi$ in $\Omega, \phi=0$ on $\partial \Omega$, the existence of which we shall assume. Assume also that $\|\phi\|_{s+2} \leqslant K\|\psi\|_{s}$. Note that (1.5) and the Sobolev imbedding theorem give the bound

$$
\begin{aligned}
\left\|a(p) \nabla \phi-\pi_{h} a(p) \nabla \phi\right\|_{0,(2+\varepsilon) / \varepsilon} & \leqslant K h^{\varepsilon / 2}\|a(p) \nabla \phi\|_{\varepsilon / 2,(2+\varepsilon) / \varepsilon} \\
& \leqslant K h^{\varepsilon / 2}\|\nabla \phi\|_{1} \leqslant K h^{\varepsilon / 2}\|\phi\|_{s+2} .
\end{aligned}
$$

Also, the Sobolev imbedding theorem implies that $a(p) \nabla \phi \in L^{t}(\Omega)^{2}$ for any finite $t$, with $\|a(p) \nabla \phi\|_{0, t} \leqslant K\|\phi\|_{2}$; the quasi-regularity of $\mathscr{T}_{h}$ implies that if $\chi \in \mathrm{V}_{h}$ and $\pi \in W_{h}$,

$$
\|\mathrm{X}\|_{0,2+\varepsilon} \leqslant K h^{-\varepsilon /(2+\varepsilon)}\|\mathrm{x}\|_{0}, \quad\|\pi\|_{0,2+\varepsilon} \leqslant K h^{-\varepsilon /(2+\varepsilon)}\|\pi\|_{0} .
$$

Let $\boldsymbol{\kappa}=\tilde{\alpha}_{p p}\left(p_{h}\right) \mathbf{u}+\tilde{\beta}_{p p}\left(p_{h}\right), \lambda=\tilde{\alpha}_{p}\left(p_{h}\right)$, and $\rho=\tilde{c}_{p p}\left(p_{h}\right)$. We have $\lambda, \rho \in L^{\infty}(\Omega)$ and $\kappa \in L^{\infty}(\Omega)^{2}$. Rewrite (2.5) as

$$
\begin{aligned}
& \text { (a) }(\alpha(p) \zeta, \mathbf{v})-(\operatorname{div} \mathbf{v}, \tau)+(\Gamma \tau, \mathbf{v}) \\
& =\left([\mathbf{k} \xi+\lambda \zeta] \xi+\Gamma\left[P_{h} p-p\right], \mathbf{v}\right), \quad \mathbf{v} \in \mathbf{V}_{h}, \\
& \text { (b) }(\operatorname{div} \zeta, w)+(\gamma \tau, w)=\left(\rho \xi^{2}+\gamma\left[P_{h} p-p\right], w\right), \quad w \in W_{h},
\end{aligned}
$$

where $\xi, \zeta$, and $\tau$ are the same as in the proof of Theorem 3.1. It follows from (1.4), (1.10), (5.3), and integration by parts (exactly as in Lemma 2.1) that

$$
\begin{aligned}
(\tau, \psi)= & \left([\kappa \xi+\lambda \zeta] \xi+\Gamma\left[P_{h} p-p\right], a(p) \nabla \phi\right) \\
& +\left([\kappa \xi+\lambda \zeta] \xi+\Gamma\left[P_{h} p-p\right], \pi_{h} a(p) \nabla \phi-a(p) \nabla \phi\right) \\
& +\left(\alpha(p) \zeta+\Gamma \tau, a(p) \nabla \phi-\pi_{h} a(p) \nabla \phi\right)+\left(\operatorname{div} \zeta+\gamma \tau, \phi-P_{h} \phi\right) \\
& +\left(\rho \xi^{2}+\gamma\left[P_{h} p-p\right], \phi\right)+\left(\rho \xi^{2}+\gamma\left[P_{h} p-p\right], P_{h} \phi-\phi\right) .
\end{aligned}
$$


First, note that (1.5), (1.8), (5.1), (5.2), and Theorem 3.1 imply

$$
\begin{aligned}
& ([\kappa \xi+\lambda \zeta] \xi, a(p) \nabla \phi) \leqslant K\left(\|\xi\|_{0,2+\varepsilon}+\|\zeta\|_{0,2+\varepsilon}\right)\|\xi\|_{0,2+\varepsilon}\|\nabla \phi\|_{0,(2+\varepsilon) / \varepsilon} \\
& \leqslant K\left(\left\|p-P_{h} p\right\|_{0,2+\varepsilon}+\|\tau\|_{0,2+\varepsilon}+\left\|\mathbf{u}-\pi_{h} \mathbf{u}\right\|_{0,2+\varepsilon}+\|\boldsymbol{\sigma}\|_{0,2+\varepsilon}\right) \\
& \times\left(\left\|p-P_{h} p\right\|_{0,2+\varepsilon}+\|\tau\|_{0,2+\varepsilon}\right)\|\nabla \phi\|_{1} \\
& \leqslant K\left(h^{r_{2}-\varepsilon /(2+\varepsilon)}\|p\|_{\left.r_{2}-\varepsilon / 2+\varepsilon\right), 2+\varepsilon}+h^{-\varepsilon /(2+\varepsilon)}\|\tau\|_{0}\right. \\
& \left.+h^{r_{2}-\varepsilon /(2+\varepsilon)}\|\mathbf{u}\|_{\left.r_{2}-\varepsilon / 2+\varepsilon\right), 2+\varepsilon}+h^{-\varepsilon /(2+\varepsilon)}\|\boldsymbol{\sigma}\|_{0}\right) \\
& \times\left(h^{l-\varepsilon /(2+\varepsilon)}\|p\|_{l-\varepsilon / 2+\varepsilon), 2+\varepsilon}+h^{-\varepsilon /(2+\varepsilon)}\|\tau\|_{0}\right)\|\phi\|_{2} \\
& \leqslant K h^{-2 \varepsilon /(2+\varepsilon)}\left(h^{r_{2}}\|p\|_{r_{2}+1}+\left\|p_{h} p-p\right\|_{0}+\|\xi\|_{0}+\left\|\pi_{h} \mathbf{u}-\mathbf{u}\right\|_{0}+\|\zeta\|_{0}\right) \\
& \times\left(h^{\prime}\|p\|_{l}+\left\|P_{h} p-p\right\|_{0}+\|\xi\|_{0}\right)\|\phi\|_{s+2} \\
& \leqslant K h^{-2 \varepsilon /(2+\varepsilon)} h^{r_{2}}\|p\|_{r_{2}+1} h^{\prime}\|p\|_{l+\delta_{0 k}}\|\phi\|_{s+2} \\
& =K h^{l+r_{2}-2 \varepsilon /(2+\varepsilon)}\|p\|_{1+\delta_{0 k}}\|p\|_{r_{2}+1}\|\phi\|_{s+2} \text {, } \\
& 2-\delta_{0 k} \leqslant l \leqslant k+1,1 \leqslant r_{2} \leqslant k+1, \\
& \left([\kappa \xi+\lambda \zeta] \xi, \pi_{h} a(p) \nabla \phi-a(p) \nabla \phi\right) \\
& \leqslant K h^{-2 \varepsilon /(2+\varepsilon)} h^{r_{2}}\|p\|_{r_{2}+1} h^{\prime}\|p\|_{l+\delta_{0 k}}\left\|\pi_{h} a(p) \nabla \phi-a(p) \nabla \phi\right\|_{0 .(2+\varepsilon) / \varepsilon} \\
& \leqslant K h^{l+r_{2}-2 \varepsilon /(2+\varepsilon)}\|p\|_{l+\delta_{0 k}}\|p\|_{r_{2}+1} h^{\varepsilon / 2}\|\phi\|_{s+2} \text {, } \\
& 2-\delta_{0 k} \leqslant l \leqslant k+1,1 \leqslant r_{2} \leqslant k+1, \\
& \left(\rho \xi^{2}, \phi\right) \leqslant K\|\xi\|_{0,2+\varepsilon}^{2}\|\phi\|_{0,(2+\varepsilon) / \varepsilon} \\
& \leqslant K h^{-2 \varepsilon /(2+\varepsilon)} h^{\prime}\|p\|_{l+\delta_{0 k}} h^{r_{2}}\|p\|_{r_{2}+\delta_{0 k}}\|\phi\|_{2} \\
& \leqslant K h^{l+r_{2}-2 \varepsilon /(2+\varepsilon)}\|p\|_{l+\delta_{0 k}}\|p\|_{r_{2}+1}\|\phi\|_{s+2}, \\
& 2-\delta_{0 k} \leqslant l \leqslant k+1,1 \leqslant r_{2} \leqslant k+1 \text {, } \\
& \left(\rho \xi^{2}, P_{h} \phi-\phi\right) \leqslant K\|\xi\|_{0,2+\varepsilon}^{2}\left\|P_{h} \phi-\phi\right\|_{0,(2+\varepsilon) / \varepsilon} \\
& \leqslant K h^{l+r_{2}-2 \varepsilon /(2+\varepsilon)}\|p\|_{l+\delta_{0 k}}\|p\|_{r_{2}+1} h^{\varepsilon / 2}\|\phi\|_{1} \\
& \leqslant K h^{l+r_{2}-2 \varepsilon /(2+\varepsilon)}\|p\|_{l+\delta_{0 k}}\|p\|_{r_{2}+1}\|\phi\|_{s+2}, \\
& 2-\delta_{0 k} \leqslant l \leqslant k+1,1 \leqslant r_{2} \leqslant k+1 .
\end{aligned}
$$

Next, we obtain from (1.9) the bound

$$
\begin{aligned}
\left(\Gamma\left[P_{h} p-p\right],\right. & a(p) \nabla \phi)+\left(\gamma\left[P_{h} p-p\right], \phi\right) \\
& \leqslant K\left\|P_{h} p-p\right\|_{-s-1}\left(\|\nabla \phi\|_{s+1}+\|\phi\|_{s+1}\right) \\
& \leqslant K h^{r_{1}+s+1}\|p\|_{r_{1}}\|\phi\|_{s+2}, \quad 0 \leqslant r_{1} \leqslant k+1 .
\end{aligned}
$$

Also, (1.5) and (1.8) give the estimates

$$
\begin{aligned}
\left(\Gamma\left[P_{h} p-p\right],\right. & \left.\pi_{h} a(p) \nabla \phi-a(p) \nabla \phi\right)+\left(\gamma\left[P_{h} p-p\right], P_{h} \phi-\phi\right) \\
& \leqslant K\left\|P_{h} p-p\right\|_{0}\left(\left\|\pi_{h} a(p) \nabla \phi-a(p) \nabla \phi\right\|_{0}+\left\|P_{h} \phi-\phi\right\|_{0}\right) \\
& \leqslant K h^{r_{1}+s+1}\|p\|_{r_{1}}\|\phi\|_{s+2}, \quad 0 \leqslant r_{1} \leqslant k+1,
\end{aligned}
$$




$$
\begin{aligned}
\left(\Gamma \tau, a(p) \nabla \phi-\pi_{h} a\right. & (p) \nabla \phi)+\left(\gamma \tau, \phi-P_{h} \phi\right) \\
& \leqslant K\|\tau\|_{0}\left(\left\|a(p) \nabla \phi-\pi_{h} a(p) \nabla \phi\right\|_{0}+\left\|\phi-P_{h} \phi\right\|_{0}\right) \\
& \leqslant K h^{s+1}\|\tau\|_{0}\|\phi\|_{s+2} .
\end{aligned}
$$

Finally, (1.5), (1.8), and Theorem 3.1 imply that

$$
\begin{aligned}
&\left(\alpha(p) \zeta, a(p) \nabla \phi-\pi_{h} a(p) \nabla \phi\right) \\
& \leqslant K\|\zeta\|_{0}\left\|a(p) \nabla \phi-\pi_{h} a(p) \nabla \phi\right\|_{0} \leqslant K h^{r_{1}+s+1}\|p\|_{r_{1}+1}\|\phi\|_{s+2}, \\
& 1 \leqslant r_{1} \leqslant k+1,
\end{aligned}
$$

$\left(\operatorname{div} \zeta, \phi-P_{h} \phi\right) \leqslant K\|\operatorname{div} \zeta\|_{0}\left\|\phi-P_{h} \phi\right\|_{0}$

$$
\leqslant K h^{r_{1}+s+1}\left\{\begin{array}{l}
\|p\|_{r_{1}+1}\|\phi\|_{s+2}, \quad 1 \leqslant r_{1} \leqslant k+2, \text { if } s \leqslant k-1, \\
\|p\|_{r_{1}+2+s-k}\|\phi\|_{s+1+k-s}, \quad 0 \leqslant r_{1} \leqslant k+1, \text { if } s>k-1 .
\end{array}\right.
$$

If we combine now (5.4)-(5.13), we see that

$$
\|\tau\|_{-s} \leqslant K\left[h^{s+1}\|\tau\|_{0}+h^{l+r_{2}-\varepsilon}\|p\|_{l+\delta_{0 k}}\|p\|_{r_{2}+1}+h^{r_{1}+s+1}\|p\|_{r_{1}+1+(s-k+1)^{+}}\right]
$$

for $2-\delta_{0 k} \leqslant l \leqslant k+1,1 \leqslant r_{1}, r_{2} \leqslant k+1$, where $K$ contains a multiple of $\|p\|_{2+\varepsilon}^{4}$. If we now take $s=0$ in (5.14), we obtain the estimate

$$
\|\tau\|_{0} \leqslant K\left[h\|\tau\|_{0}+h^{r_{2}+l-\varepsilon}\|p\|_{l+\delta_{0 k}}\|p\|_{r_{2}+1}+h^{r_{1}+1}\|p\|_{r_{1}+1+\delta_{0 k}}\right],
$$

which, for sufficiently small $h$, implies that

$$
\|\tau\|_{0} \leqslant K\left[h^{l+r_{2}-\varepsilon}\|p\|_{l+\delta_{0 k}}\|p\|_{r_{2}+1}+h^{r_{1}+1}\|p\|_{r_{1}+1+\delta_{0 k}}\right] .
$$

Substituting (5.15) into (5.14) completes the proof of the lemma. Q.E.D.

We can now demonstrate the convergence of $p_{h}$ to $p$ to be at an optimal rate in $L^{q}(\Omega), 2 \leqslant q \leqslant \infty$ (for $k>0$ if $q>2 / \varepsilon$ ).

THEOREM 5.1. There are positive constants $C_{q}$ and $C$ (both containing a multiple of $\left.\|p\|_{2+\varepsilon}^{4}\right)$, independent of $h$, such that, if $p \in W^{r, q}(\Omega) \cap H^{r+1+\delta_{0 k}+t(q, r)}(\Omega), 1 \leqslant r$ $\leqslant k+1-(\varepsilon-2 / q)^{+} \delta_{0 k}$, where $t(q, r)=-(2 / q)+(1+(2 / q)-r)^{+}$, then

$$
\begin{aligned}
& \text { (a) }\left\|p-p_{h}\right\|_{0, q} \leqslant C_{q} h^{r}\|p\|_{r+1+\delta_{0 k}+t(q, r)}, \quad q<\infty \text {, } \\
& \text { (b) }\left\|p-p_{h}\right\|_{0, \infty} \leqslant C h^{r}\left[\|p\|_{r, \infty}+\|p\|_{r+1+\delta_{0 k}}\right] .
\end{aligned}
$$

Proof. Using Lemma 5.1 (with $s=0, l=1+\varepsilon\left(1-\delta_{0 k}\right), r_{1}+2 / q=r_{2}+(2 / q)$ $\left.-\varepsilon \delta_{0 k}=r\right),(1.8)$, and the quasi-regularity of $\mathscr{T}_{h}$, we obtain the bounds

(a) $\left\|p-p_{h}\right\|_{0, q} \leqslant\left\|p-P_{h} p\right\|_{0, q}+\|\tau\|_{0, q}$

$$
\begin{aligned}
& \leqslant K_{q}\left[h^{r}\|p\|_{r, q}+h^{-(q-2) / q}\|\tau\|_{0}\right] \\
& \leqslant C_{q}\left[h^{r}\|p\|_{r, q}+h^{r}\|p\|_{r+1+\delta_{0 k}+t(q, r)^{+}}\right],
\end{aligned}
$$

(b) $\left\|p-p_{h}\right\|_{0, \infty} \leqslant\left\|p-P_{h} p\right\|_{0, \infty}+\|\tau\|_{0, \infty}$

$$
\begin{aligned}
& \leqslant K\left[h^{r}\|p\|_{r, \infty}+h^{-1}\|\tau\|_{0}\right] \\
& \leqslant K\left[h^{r}\|p\|_{r, \infty}+h^{-1+r+1}\|p\|_{r+1+\delta_{0 k}}\right] \text {. Q.E.D. }
\end{aligned}
$$


6. Error Estimates in $H^{s}(\Omega)^{\prime}$ and $\left(H^{s}(\Omega)^{2}\right)^{\prime}$. We can also derive negative norm error estimates from Lemma 5.1.

THEOREM 6.1. There exists a constant $C>0$ (containing a multiple of $\|p\|_{2+\varepsilon}^{4}$ ), independent of $h$, such that, if $p$ is sufficiently smooth, then for $0 \leqslant s \leqslant k+1$ we have the following estimates:

(i) $\left\|p-p_{h}\right\|_{-s} \leqslant C\left[h^{r_{1}+s}\|p\|_{r_{1}+(s-k+1)^{+}}+h^{r_{2}+l-\varepsilon}\|p\|_{l+\delta_{0 k}}\|p\|_{r_{2}+1}\right]$,

$$
2-\delta_{0 k} \leqslant l \leqslant k+1,1 \leqslant r_{1}, r_{2} \leqslant k+1 \text {, }
$$

(ii) $\left\|\mathbf{u}-\mathbf{u}_{h}\right\|_{-s} \leqslant C\left[h^{r_{1}+s}\|p\|_{r_{1}+1+(s-k)^{+}}+h^{r_{2}+l-\varepsilon}\|p\|_{l+\varepsilon+\delta_{0 k}}\|p\|_{r_{2}+1+\varepsilon \delta_{0 k}}\right]$,

$$
2-\delta_{0 k} \leqslant l \leqslant k+1,1 \leqslant r_{1}, r_{2} \leqslant k+1,
$$

(iii) $\left\|\operatorname{div}\left(\mathbf{u}-\mathbf{u}_{h}\right)\right\|_{-s} \leqslant C\left[h^{r_{1}+s}\|p\|_{r_{1}+2}+h^{r_{2}+l-\varepsilon}\|p\|_{l+\varepsilon+\delta_{0 k}}\|p\|_{r_{2}+1+\varepsilon \delta_{0 k}}\right]$,

$$
2-\delta_{0 k} \leqslant l \leqslant k+1,0 \leqslant r_{1}, r_{2} \leqslant k+1 \text {. }
$$

Proof. (i) follows directly from Lemma 5.1 and (1.9), since

$$
\xi=\tau+\left(P_{h} p-p\right), \quad\left(\|\xi\|_{-k-1} \leqslant\|\tau\|_{-k}+\left\|P_{h} p-p\right\|_{-k-1}\right)
$$

Rewrite (5.3) as

$$
\left\{\begin{array}{l}
(\alpha(p) \zeta, \mathbf{v})=(\operatorname{div} \mathbf{v}, \tau)-(\Gamma \xi, \mathbf{v})+([\kappa \xi+\lambda \zeta] \xi, \mathbf{v}), \quad \mathbf{v} \in \mathbf{V}_{h} \\
(\operatorname{div} \zeta, w)=(-\gamma \xi, w)+\left(\rho \xi^{2}, w\right), \quad w \in W_{h}
\end{array}\right.
$$

Note that (iii) for $s=0$ is just a consequence of (iii) of Theorem 3.1. Let then $\psi \in H^{s}(\Omega), 1 \leqslant s \leqslant k+1$. Then, $\psi \in L^{\theta / 2}(\Omega)$ with $\|\psi\|_{\theta / 2} \leqslant K\|\psi\|_{s}$. The second equation of (6.1) and (1.8) imply that

$$
\begin{aligned}
(\operatorname{div} \zeta, \psi)= & \left(\operatorname{div} \zeta, P_{h} \psi\right)+\left(\operatorname{div} \zeta, \psi-P_{h} \psi\right) \\
= & -\left(\gamma \xi, P_{h} \psi\right)+\left(\rho \xi^{2}, P_{h} \psi\right)+\left(\operatorname{div} \zeta, \psi-P_{h} \psi\right) \\
= & -(\gamma \xi, \psi)+\left(\gamma \xi, \psi-P_{h} \psi\right)+\left(\rho \xi^{2}, \psi\right)+\left(\rho \xi^{2}, P_{h} \psi-\psi\right) \\
& +\left(\operatorname{div} \zeta, \psi-P_{h} \psi\right) \\
\leqslant & K\|\psi\|_{s}\left[\|\xi\|_{-s}+h^{s}\|\xi\|_{0}+\|\xi\|_{0,2+\varepsilon}^{2}+h^{s}\|\xi\|_{0,4}^{2}+h^{s}\|\operatorname{div} \zeta\|_{0}\right]
\end{aligned}
$$

since $[1 /(2+\varepsilon)]+[1 /(2+\varepsilon)]+[1 /(\theta / 2)]=1$. Therefore, (iii) follows from (i), (a) of Theorem 5.1, (iii) of Theorem 3.1, and interpolation [10] for $0<s<1$.

Finally, assume that the coefficient $a$ is smooth enough that for $\chi \in H^{s-1}(\Omega)$ there is a unique $\phi \in H^{s+1}(\Omega)$ such that $\nabla \cdot(a(p) \nabla \phi)=\chi$ in $\Omega, \phi=0$ on $\partial \Omega$, with $\|\phi\|_{s+1} \leqslant K\|\chi\|_{s-1}, 0 \leqslant s \leqslant k+1$. Let $\psi \in H^{s}(\Omega)^{2}$; let $\phi \in H^{s+1}(\Omega)$ be the solution of $\nabla(a(p) \nabla \phi)=\operatorname{div} \psi$ in $\Omega, \phi=0$ on $\partial \Omega$, and let $\chi=\psi-a(p) \nabla \phi$. Then $\operatorname{div} \mathrm{\chi}=0,\|\mathrm{x}\|_{s} \leqslant K\|\psi\|_{s}$, and $\|\phi\|_{s+1} \leqslant K\|\psi\|_{s}$. It follows from (1.4), (1.5), (1.3), 
(1.8), (1.10), (5.1), integration by parts, Theorem 3.1 and Theorem 5.1 that

$$
\begin{aligned}
& (\alpha(p) \zeta, \psi)=(\alpha(p) \zeta, \chi)+(\zeta, \nabla \phi) \\
& =-(\operatorname{div} \zeta, \phi)+\left(\alpha(p) \zeta, \pi_{h} \mathrm{X}\right)+\left(\alpha(p) \zeta, \mathrm{X}-\pi_{h} \mathrm{X}\right) \\
& =-\left(\operatorname{div} \zeta, P_{h} \phi\right)+\left(\operatorname{div} \zeta, P_{h} \phi-\phi\right)+\left(\alpha(p) \zeta, \chi-\pi_{h} \mathrm{X}\right)+\left(\alpha(p) \zeta, \pi_{h} \mathrm{X}\right) \\
& =\left(\gamma \xi, P_{h} \phi\right)-\left(\rho \xi^{2}, P_{h} \phi\right)+\left(\operatorname{div} \zeta, P_{h} \phi-\phi\right)+\left(\alpha(p) \zeta, \chi-\pi_{h} \chi\right) \\
& -\left(\Gamma \xi, \pi_{h} \mathrm{X}\right)+\left([\kappa \xi+\lambda \xi] \xi, \pi_{h} \mathrm{X}\right) \\
& =(\gamma \xi, \phi)+\left(\gamma \xi, P_{h} \phi-\phi\right)-\left(\rho \xi^{2}, \phi\right)-\left(\rho \xi^{2}, P_{h} \phi-\phi\right) \\
& +\left(\operatorname{div} \zeta, P_{h} \phi-\phi\right)+\left(\alpha(p) \zeta, \chi-\pi_{h} \chi\right)-(\Gamma \xi, \chi) \\
& -\left(\Gamma \xi, \pi_{h} \chi-\chi\right)+([\kappa \xi+\lambda \xi] \xi, x)+\left([\kappa \xi+\lambda \zeta] \xi, \pi_{h} \chi-\chi\right) \\
& \leqslant K\|\psi\|_{s}\left[\|\xi\|_{-s-1}+h^{s+1}\|\xi\|_{0}+\|\xi\|_{0,4}^{2}+h^{s+1}\|\xi\|_{0,4}^{2}\right. \\
& +h^{s+1}\|\operatorname{div} \zeta\|_{0}+h^{s}\|\zeta\|_{0}+\|\xi\|_{-s}+h^{s}\|\xi\|_{0} \\
& \left.+\left(\|\zeta\|_{0,2+\varepsilon}+\|\xi\|_{0,2+\varepsilon}\right)\|\xi\|_{0,(2+\varepsilon) / \varepsilon}+h^{s}\left(\|\zeta\|_{0}+\|\xi\|_{0}\right)\|\xi\|_{0, \infty}\right] \\
& \leqslant K\|\psi\|_{s}\left[h^{r_{2}+l-\varepsilon}\|p\|_{r_{2}+1+\varepsilon \delta_{0 k}}\|p\|_{l+\varepsilon+\delta_{0 k}}+h^{r_{1}+s}\|p\|_{r_{1}+1+(s-k)^{+}}\right] \text {, }
\end{aligned}
$$

from which (ii) follows immediately. Q.E.D.

Department of Mathematics

University of Chicago

Chicago, Illinois 60637

1. S. Agmon, A. Douglis \& L. Nirenberg, "Estimates near the boundary for solutions of elliptic partial differential equations satisfying general boundary conditions," Comm. Pure Appl. Math., v. 12, 1959 , pp. 623-727.

2. F. BREZZI, "On the existence, uniqueness and approximation of saddle-point problems arising from Lagrangian multipliers," RAIRO Anal. Numér., v. 2, 1974, pp. 129-151.

3. P. Ciarlet, The Finite Element Method for Elliptic Problems, North-Holland, Amsterdam, 1978.

4. J. Douglas, JR., " $H^{1}$-Galerkin methods for a nonlinear Dirichlet problem," in Proc. Conf. on Mathematical Aspects of Finite Element Methods, Lecture Notes in Math., Vol. 606, Springer-Verlag, Berlin, 1977, pp. 64-86.

5. J. Douglas, JR. \& J. E. Roberts, "Mixed finite element methods for second order elliptic problems," Mat. Apl. Comput., v. 1, 1982, pp. 91-103.

6. J. Douglas, JR. \& J. E. Roberts, "Global estimates for mixed methods for second order elliptic equations," Math. Comp., v. 44, 1985, pp. 39-52.

7. R. S. FAlK \& J. E. OsBoRn, "Error estimates for mixed methods," RAIRO Anal. Numér., v. 14. 1980, pp. $249-277$.

8. D. Gilbarg \& N. Trudinger, Elliptic Partial Differential Equations of Second Order, Grundlehren der Mathematischen Wissenschaften, Vol. 224, Springer-Verlag, Berlin, 1977.

9. C. JoHNSON \& V. THOMEE, "Error estimates for some mixed finite element methods for parabolic type problems," RAIRO Anal. Numer., v. 15, 1981, pp. 41-78.

10. J. L. Lions \& E. Magenes, Non Homogeneous Boundary Value Problems and Applications, I, Springer-Verlag, Berlin, 1970

11. J. C. NeDELEC, “Mixed finite elements in $R^{3}$," Numer. Math., v. 35, 1980, pp. 315-341.

12. P. A. Raviart \& J. M. Thomas, "A mixed finite element method for 2 nd order elliptic problems," in Proc. Conf. on Mathematical Aspects of Finite Element Methods, Lecture Notes in Math., Vol. 606, Springer-Verlag, Berlin, 1977, pp. 292-315. 
13. R. SCHOLz, " $L_{\infty}$-convergence of saddle-point approximations for second order problems," $R A I R O$ Anal. Numér., v. 11, 1977, pp.209-216.

14. R. Scholz, "A remark on the rate of convergence for a mixed finite element method for second order elliptic problems,” Numer. Funct. Anal. Optim., v. 4, 1981-1982, pp. 269-277.

15.R. SCHOLz, "Optimal $L_{\infty}$-estimates for a mixed finite element method for second order elliptic and parabolic problems," Calcolo, v. 20, 1983, pp. 355-377.

16. J. M. ThомаS, Sur l'Analyse Numérique des Méthodes d'Éléments Finis Hybrides et Mixtes, Thèse, Université P. et M. Curie, Paris, 1977.

17. H. Triebel, Interpolation Theory, Function Spaces, Differential Operators, North-Holland, Amsterdam, 1978. 